\title{
Neural-Network-Based Approach for Extracting Eigenvectors and Eigenvalues of Real Normal Matrices and Some Extension to Real Matrices
}

\author{
Xiongfei Zou, ${ }^{1}$ Ying Tang, ${ }^{2}$ Shirong Bu, ${ }^{1}$ Zhengxiang Luo, ${ }^{1}$ and Shouming Zhong ${ }^{3}$ \\ ${ }^{1}$ School of Optoelectronic Information, University of Electronic Science and Technology of China, Chengdu 610054, China \\ ${ }^{2}$ School of Computer Science and Engineering, University of Electronic Science and Technology of China, Chengdu 611731, China \\ ${ }^{3}$ School of Mathematical Sciences, University of Electronic Science and Technology of China, Chengdu 611731, China
}

Correspondence should be addressed to Xiongfei Zou; zouxiongfei@sohu.com

Received 30 October 2012; Accepted 16 January 2013

Academic Editor: Nicola Mastronardi

Copyright (C) 2013 Xiongfei Zou et al. This is an open access article distributed under the Creative Commons Attribution License, which permits unrestricted use, distribution, and reproduction in any medium, provided the original work is properly cited.

This paper introduces a novel neural-network-based approach for extracting some eigenpairs of real normal matrices of order $n$. Based on the proposed algorithm, the eigenvalues that have the largest and smallest modulus, real parts, or absolute values of imaginary parts can be extracted, respectively, as well as the corresponding eigenvectors. Although the ordinary differential equation on which our proposed algorithm is built is only $n$-dimensional, it can succeed to extract $n$-dimensional complex eigenvectors that are indeed $2 n$-dimensional real vectors. Moreover, we show that extracting eigen-pairs of general real matrices can be reduced to those of real normal matrices by employing the norm-reducing skill. Numerical experiments verified the computational capability of the proposed algorithm.

\section{Introduction}

The problem of extracting special eigenpairs of real matrices has attracted much attention both in theory [1-4] and in many engineering fields such as real-time signal processing [5-8] and principal or minor component analysis [9-12]. For example, we may wish to get eigenvectors and the corresponding eigenvalues that (1) have the largest or smallest modulus; (2) have the largest or smallest real parts; (3) have the largest or smallest imaginary parts in absolute value. Two most popular methods for this problem are the power method and the Rayleigh quotient method in their direct forms or in the context of inverse iteration [13]. Recently, many neuralnetwork-based methods have also been proposed to solve this problem [14-23]. However, most of those neural network based methods focused on computing eigenpairs of real symmetric matrices. The following two ordinary differential equations (ODEs):

$$
\begin{gathered}
\frac{\mathrm{d} x(t)}{\mathrm{d} t}=A x(t)-x(t)^{T} A x(t) x(t), \\
\frac{\mathrm{d} x(t)}{\mathrm{d} t}=x(t)^{T} x(t) A x(t)-x(t)^{T} A x(t) x(t)
\end{gathered}
$$

were proposed by $[19,23]$, respectively, where $A$ is a real symmetric matrix. Both (1) and (2) are efficient to compute the largest eigenvalue of $A$, as well as the corresponding eigenvector. In addition, they can succeed to compute the smallest eigenvalue of $A$ and the corresponding eigenvector by simply replacing $A$ with $-A$, for example,

$$
\frac{\mathrm{d} x(t)}{\mathrm{d} t}=-x(t)^{T} x(t) A x(t)+x(t)^{T} A x(t) x(t) .
$$

The following ODE for solving the generalized eigenvalue problem was proposed by [22]

$$
\frac{\mathrm{d} x(t)}{\mathrm{d} t}=A x(t)-f(x(t)) B x(t),
$$

where $A$ and $B$ are two real symmetric matrices, and $f$ can be a general form to some degree. Particularly, if $B$ is the identity matrix, (4) can be used to solve the standard eigenvalue problem as (1) and (2).

References [16-18] extended those neural network based approaches to the case of real antisymmetric or special real matrices of order $n$, where the proposed neural networks can 
be summarized by $2 n$-dimensional ODEs since eigenvectors in those cases may be $n$-dimensional complex vectors, that is, $2 n$-dimensional real vectors.

In this paper, we propose an approach for extracting six types of eigenvalues of $n$-by- $n$ real normal matrices and the corresponding eigenvectors based on (2) or (3). Although eigenvectors of real normal matrices may be $n$-dimensional complex vectors, the computation of our proposed method can be achieved in $n$-dimensional real vector space, which can reduce the scale of networks a lot. Then, we show that any real matrix can be made arbitrarily close to a normal matrix by a series of similarity transformations, based on which our proposed algorithm can be extended to the case of arbitrary real matrices.

\section{Main Results}

Let $i=\sqrt{-1}$ be the imaginary unit, $\bar{x}$ the conjugate of $x$, and $\operatorname{diag}\left[A_{1}, \ldots, A_{p}\right]$ a block diagonal matrix, where $A_{i}, i=$ $1, \ldots, p$, is a square matrix at the $i$ th diagonal block. Unless specially stated, $A$ is a real normal matrix in this paper.

Lemma 1. In [13], $A$ is a real normal matrix of order $n$ if and only if there exists an orthogonal matrix $U$ such that

$$
\begin{gathered}
U^{T} A U=\operatorname{diag}[\underbrace{\lambda_{1}, \ldots, \lambda_{r}}_{r}, \underbrace{A_{r+1}, \ldots, A_{r+k}}_{k}], \\
r+2 k=n, \quad 0 \leq r \leq n,
\end{gathered}
$$

where

$$
\begin{aligned}
& U=[\underbrace{u_{1}, \ldots, u_{r}}_{r \text { real eigenvectors }}, \underbrace{u_{r+1}^{R}, u_{r+1}^{I}, \ldots, u_{r+k}^{R}, u_{r+k}^{I}}_{k \text { pairs of complex eigenvectors }}], \\
& A_{r+j}=\left(\begin{array}{cc}
a_{r+j} & \left|b_{r+j}\right| \\
-\left|b_{r+j}\right| & a_{r+j}
\end{array}\right), \quad j=1, \ldots, k .
\end{aligned}
$$

Here, $\lambda_{j}, j=1, \ldots, r$, are $r$ real eigenvalues of $A$ corresponding to the real eigenvectors $u_{j}$, and $a_{r+s} \pm i b_{r+s}, s=1, \ldots, k$, are $k$ pairs of complex eigenvalues of $A$ corresponding to the $k$ pairs of complex eigenvectors $u_{r+s}^{R} \pm i u_{r+s}^{I}$.

For simplicity, let $a_{j}=\lambda_{j}, b_{j}=0$ for $j=1, \ldots, r$. Based on (5) and (6), it is straightforward to verify

$$
\begin{aligned}
& U^{T}\left(A-A^{T}\right)^{T}\left(A-A^{T}\right) U \\
& =4 \operatorname{diag}\left[b_{1}^{2}, \ldots, b_{r}^{2}, b_{r+1}^{2}, b_{r+1}^{2}, \ldots, b_{r+k}^{2}, b_{r+k}^{2}\right] \\
& U^{T}\left(A+A^{T}\right) U \\
& \quad=2 \operatorname{diag}\left[a_{1}, \ldots, a_{r}, a_{r+1}, a_{r+1}, \ldots, a_{r+k}, a_{r+k}\right] \\
& U^{T}\left(A A^{T}\right) U \\
& =\operatorname{diag}\left[a_{1}^{2}, \ldots, a_{r}^{2}, a_{r+1}^{2}+b_{r+1}^{2}, a_{r+1}^{2}\right. \\
& \left.\quad+b_{r+1}^{2}, \ldots, a_{r+k}^{2}+b_{r+k}^{2}, a_{r+k}^{2}+b_{r+k}^{2}\right] .
\end{aligned}
$$
eigenvector of $-\left(A-A^{T}\right)^{2}$ corresponding to $4 b_{r+k}^{2}$ and $4 b_{r+k}^{2}=$ $\lim _{t \rightarrow \infty}\left(-x(t)^{T}\left(A-A^{T}\right)^{2} x(t) / x(t)^{T} x(t)\right)$ (thus, $\left|b_{r+k}\right|$ has been gotten), where $x(t)$ is a solution of (10). If $b_{r+k}=0, b_{j}$ 's
Then, the following six definitions and two lemmas are presented, which will be involved much in the sequel.

(C1) Let $\mathscr{J}_{1}=\left\{s|| b_{s} \mid=\max \left\{\left|b_{1}\right|, \ldots,\left|b_{r+k}\right|\right\}\right.$. Then for all $j, k \in \mathscr{J}_{1}, a_{j}=a_{k}$.

(C2) Let $\mathscr{J}_{2}=\left\{s|| b_{s} \mid=\min \left\{\left|b_{1}\right|, \ldots,\left|b_{r+k}\right|\right\}\right.$. Then for all $j, k \in \mathscr{J}_{2}, a_{j}=a_{k}$.

(C3) Let $\mathscr{J}_{3}=\left\{s \mid a_{s}=\max \left\{a_{1}, \ldots, a_{r+k}\right\}\right.$. Then for all $j, k \in \mathscr{J}_{3},\left|b_{j}\right|=\left|b_{k}\right|$.

(C4) Let $\mathscr{J}_{4}=\left\{s \mid a_{s}=\min \left\{a_{1}, \ldots, a_{r+k}\right\}\right.$. Then for all $j, k \in \mathscr{J}_{4},\left|b_{j}\right|=\left|b_{k}\right|$.

(C5) Let $\mathscr{J}_{5}=\left\{s \mid a_{s}^{2}+b_{s}^{2}=\max \left\{a_{1}^{2}+b_{1}^{2}, \ldots, a_{r+k}^{2}+b_{r+k}^{2}\right\}\right.$. Then for all $j, k \in \mathscr{J}_{5}, a_{j}=a_{k}$.

(C6) Let $\mathscr{J}_{6}=\left\{s \mid a_{s}^{2}+b_{s}^{2}=\min \left\{a_{1}^{2}+b_{1}^{2}, \ldots, a_{r+k}^{2}+b_{r+k}^{2}\right\}\right.$. Then for all $j, k \in \mathscr{J}_{6}, a_{j}=a_{k}$.

Lemma 2 (Theorem 4 in [23]). Assume that nonzero $x(0) \epsilon$ $R^{n}$ is not orthogonal to the eigensubspace corresponding to the largest eigenvalue of $A$. Then, the solution of (2) starting from $x(0)$ converges to an eigenvector corresponding to the largest eigenvalue of $A$ that is equal to $\lim _{t \rightarrow+\infty}\left(x(t)^{T} A x(t) / x(t)^{T} x(t)\right)$.

Lemma 3 (Theorem 5 in [23]). Assume that nonzero $x(0) \epsilon$ $R^{n}$ is not orthogonal to the eigensubspace corresponding to the smallest eigenvalue of $A$. Then, the solution of (3) starting from $x(0)$ converges to an eigenvector corresponding to the smallest eigenvalue of $A$ that is equal to $\lim _{t \rightarrow+\infty}\left(x(t)^{T} A x(t) / x(t)^{T} x(t)\right)$.

Remark 4. If we randomly choose $x(0)$, the projection of $x(0)$ on the eigensubspace corresponding to the largest or smallest eigenvalue of $A$ will be nonzero with high probability. Hence, (2) and (3) can almost work well with randomly generated $x(0)$.

2.1. Computing the Eigenvalues with the Largest or Smallest Imaginary Parts in Absolute Value, as well as the Corresponding Eigenvectors. Without loss of generality, in this subsection we assume that $b_{1}=\cdots=b_{r}=0<b_{r+1}^{2} \leq \cdots \leq b_{r+k}^{2}$ in (7). Note that $\left(A-A^{T}\right)^{T}\left(A-A^{T}\right)=-\left(A-A^{T}\right)^{2}$.

Based on (7), we know that 0 (if any) is the eigenvalue of $-\left(A-A^{T}\right)^{2}$ corresponding to the eigenvector $u_{j}, j=1, \ldots, r$, and that $4 b_{r+s}^{2}, s=1, \ldots, k$, is the eigenvalue of $-\left(A-A^{T}\right)^{2}$ corresponding to the eigenvectors $u_{r+s}^{R}$ or $u_{r+s}^{I}$. If replacing $A$ with the symmetric matrix $-\left(A-A^{T}\right)^{2}$ in (2) as

$$
\begin{aligned}
\frac{\mathrm{d} x(t)}{\mathrm{d} t}= & -x(t)^{T} x(t)\left(A-A^{T}\right)^{2} x(t) \\
& +x(t)^{T}\left(A-A^{T}\right)^{2} x(t) x(t)
\end{aligned}
$$

based on Lemma 2, we know that $\alpha=\lim _{t \rightarrow \infty} x(t)$ is an 
are all zero, meaning that $A$ is symmetric. In this case, we can directly use (2) or (3) to get the largest or smallest eigenvalue of $A$ and the corresponding eigenvector, respectively. Hence, we assume $b_{r+k} \neq 0$.

The following lemma introduces an approach for computing $a_{r+k}$ under the condition (C1).

Lemma 5. Assume that (C1) holds. Let $\alpha=\lim _{t \rightarrow \infty} x(t)$, where $x(t)$ is a solution of (10). Then, $a_{r+k}=\alpha^{T}\left(A+A^{T}\right) \alpha /$ $2 \alpha^{T} \alpha$. In addition, there exists $a_{r+k}^{2}+b_{r+k}^{2}=\alpha^{T}\left(A A^{T}\right) \alpha / \alpha^{T} \alpha$.

Proof. Assume that $4 b_{r+j}^{2}, j=s, s+1, \ldots, k, s \geq 1$, are the largest eigenvalues of $-\left(A-A^{T}\right)^{2}$, that is; $\left|b_{r+s}\right|=\left|b_{r+s+1}\right|=$ $\cdots=\left|b_{r+k}\right|$. Therefore,

$$
\mathscr{J}_{1}=\{r+s, \ldots, r+k\} .
$$

Based on Lemma 2 and (7), we know that $\alpha$ should be a linear combination of $u_{j}^{R}$ and $u_{j}^{I}, j \in \mathscr{J}_{1}$. Let

$$
\alpha=\sum_{j \in \mathscr{F}_{1}}\left(\gamma_{j} u_{j}^{R}+\tau_{j} u_{j}^{I}\right), \quad \gamma_{j}, \tau_{j} \in R
$$

In addition, by (8) we have

$$
\left(A+A^{T}\right) u_{j}^{R}=2 a_{j} u_{j}^{R}, \quad\left(A+A^{T}\right) u_{j}^{I}=2 a_{j} u_{j}^{I}, \quad j \in \mathscr{J}_{1} .
$$

And by (9), we have

$$
\begin{gathered}
\left(A A^{T}\right) u_{j}^{R}=\left(a_{j}^{2}+b_{j}^{2}\right) u_{j}^{R}, \\
\left(A A^{T}\right) u_{j}^{I}=\left(a_{j}^{2}+b_{j}^{2}\right) u_{j}^{I}, \quad j \in \mathscr{J}_{1} .
\end{gathered}
$$

Because $U$ is an orthogonal matrix and for all $j \in \mathscr{J}_{1}$, $a_{j}=a_{r+k}$ holds due to $(\mathrm{C} 1)$, it is straightforward to verify

$$
\begin{gathered}
\frac{\alpha^{T}\left(A+A^{T}\right) \alpha}{2 \alpha^{T} \alpha}=\frac{2 a_{r+k} \sum_{j \in \mathscr{F}_{1}}\left(\gamma_{j}^{2}+\tau_{j}^{2}\right)}{2 \sum_{j \in \mathscr{F}_{1}}\left(\gamma_{j}^{2}+\tau_{j}^{2}\right)}=a_{r+k}, \\
\frac{\alpha^{T}\left(A A^{T}\right) \alpha}{\alpha^{T} \alpha}=\frac{\left(a_{r+k}^{2}+b_{r+k}^{2}\right) \sum_{j \in \mathscr{F}_{1}}\left(\gamma_{j}^{2}+\tau_{j}^{2}\right)}{\sum_{j \in \mathscr{F}_{1}}\left(\gamma_{j}^{2}+\tau_{j}^{2}\right)}=a_{r+k}^{2}+b_{r+k}^{2},
\end{gathered}
$$

thus proving the lemma.

Remark 6. Based on Lemma 5, if $a_{r+k}^{2}+b_{r+k}^{2} \neq \alpha^{T}\left(A A^{T}\right) \alpha / \alpha^{T} \alpha$, (C1) does not hold surely, which can be used to check whether (C1) holds or not.

The following lemma introduces an approach for computing a pair of conjugated eigenvectors of $A$ corresponding to the eigenvalues $a_{r+k} \pm i\left|b_{r+k}\right|$ under the condition (C1).

Lemma 7. Assume that (C1) holds. Given any nonzero $4 b_{r+k}^{2}$, the largest eigenvalue of $-\left(A-A^{T}\right)^{2}$, and the corresponding eigenvector $\alpha$ obtained by (10), let $a_{r+k}=\alpha^{T}\left(A+A^{T}\right) \alpha / 2 \alpha^{T} \alpha$ and $\beta=\left(A-A^{T}\right) \alpha / 2\left|b_{r+k}\right|$. Then, $a_{r+k} \pm i\left|b_{r+k}\right|$ are two eigenvalues of $A$ corresponding to the eigenvectors $\beta \pm i \alpha$, respectively.
Proof. Let $\mathscr{J}_{1}$ and $\alpha$ take the form as (11) and (12), respectively. Based on (13), we can write

$$
\frac{\left(A+A^{T}\right)}{2} \alpha=a_{r+k} \alpha .
$$

Following the decomposition of $A$ as (5) and the definition of $\beta$, we have

$$
\begin{aligned}
\left|b_{r+k}\right| \beta & =\left(A-A^{T}\right) \frac{\alpha}{2} \\
& =U^{T} \operatorname{diag}[\underbrace{0, \ldots, 0}_{r}, B_{r+1}, \ldots, B_{r+k}] U \frac{\alpha}{2},
\end{aligned}
$$

where

$$
B_{j}= \begin{cases}\left(\begin{array}{cc}
0 & 2\left|b_{j}\right| \\
-2\left|b_{j}\right| & 0
\end{array}\right), & j \in\{r+1, \ldots, r+k\} \backslash \mathscr{J}_{1} \\
\left(\begin{array}{cc}
0 & 2\left|b_{r+k}\right| \\
-2\left|b_{r+k}\right| & 0
\end{array}\right), & j \in \mathscr{J}_{1} .\end{cases}
$$

Taking (12) into (17), we get

$$
\beta=\sum_{j \in \mathscr{F}_{1}}\left(\tau_{j} u_{j}^{R}-\gamma_{j} u_{j}^{I}\right) .
$$

Based on (13) and (19), it is straightforward to verify

$$
\frac{\left(A+A^{T}\right)}{2} \beta=a_{r+k} \beta .
$$

In addition, since $4 b_{r+k}^{2}$ is the eigenvalue of $-\left(A-A^{T}\right)^{2}$ corresponding to the eigenvector $\alpha$, we have $-\left(A-A^{T}\right)^{2} \alpha=$ $4 b_{r+k}^{2} \alpha$. Hence, $-\left(A-A^{T}\right)\left|b_{r+k}\right| \beta=2 b_{r+k}^{2} \alpha$. Since $b_{r+k} \neq 0$, we have

$$
\frac{\left(A-A^{T}\right)}{2} \beta=-\left|b_{r+k}\right| \alpha .
$$

By (20) and (21), we have

$$
A \beta=\frac{A+A^{T}}{2} \beta+\frac{A-A^{T}}{2} \beta=a_{r+k} \beta-\left|b_{r+k}\right| \alpha .
$$

By (16) and (17), we have

$$
A \alpha=\frac{A+A^{T}}{2} \alpha+\frac{A-A^{T}}{2} \alpha=a_{r+k} \alpha+\left|b_{r+k}\right| \beta .
$$

Then, it is straightforward to verify

$$
\begin{aligned}
& A(\beta+i \alpha)=\left(a_{r+k}+i\left|b_{r+k}\right|\right)(\beta+i \alpha), \\
& A(\beta-i \alpha)=\left(a_{r+k}-i\left|b_{r+k}\right|\right)(\beta-i \alpha),
\end{aligned}
$$

thus proving the lemma. 
To get $\left|b_{1}\right|$, we can in advance get $4 b_{1}^{2}$, the smallest eigenvalue of $-\left(A-A^{T}\right)^{2}$ by replacing $A$ with $-\left(A-A^{T}\right)^{2}$ in (3) as follows:

$$
\begin{aligned}
\frac{\mathrm{d} x(t)}{\mathrm{d} t}= & x(t)^{T} x(t)\left(A-A^{T}\right)^{2} x(t) \\
& -x(t)^{T}\left(A-A^{T}\right)^{2} x(t) x(t) .
\end{aligned}
$$

Let $\alpha^{*}=\lim _{t \rightarrow \infty} x(t)$, where $x(t)$ is a solution of (25). From Lemma 3, we know $4 b_{1}^{2}=-\left(\alpha^{*}\right)^{T}\left(A-A^{T}\right)^{2} \alpha^{*} /\left(\alpha^{*}\right)^{T} \alpha^{*}$. Then, the following lemma similar to Lemma 5 can be used to compute $a_{1}$.

Lemma 8. Assume that (C2) holds. Then, $a_{1}=\left(\alpha^{*}\right)^{T}(A+$ $\left.A^{T}\right) \alpha^{*} / 2\left(\alpha^{*}\right)^{T} \alpha^{*}$. In addition, there exists $a_{1}^{2}+b_{1}^{2}=$ $\left(\alpha^{*}\right)^{T}\left(A A^{T}\right) \alpha^{*} /\left(\alpha^{*}\right)^{T} \alpha^{*}$.

Proof. The proof is almost the same to that in Lemma 5.

Note that $b_{1}$ may be zero; that is, $-\left(A-A^{T}\right)^{2}$ has real eigenvalues. In this case, we have the following lemma.

Lemma 9. Assume that (C2) holds and 0 is the smallest eigenvalue of $-\left(A-A^{T}\right)^{2}$ corresponding to the eigenvector $\alpha^{*}$. Then, $a_{1}$ is the eigenvalue of $A$ corresponding to the eigenvector $\alpha^{*}$, where $a_{1}=\left(\alpha^{*}\right)^{T}\left(A+A^{T}\right) \alpha^{*} / 2\left(\alpha^{*}\right)^{T} \alpha^{*}$.

Proof. Following the conditions, we have $-\left(A-A^{T}\right)^{2} \alpha^{*}=0$. Hence, $\left(\alpha^{*}\right)^{T}\left(A-A^{T}\right)^{T}\left(A-A^{T}\right) \alpha^{*}=\left\|\left(A-A^{T}\right) \alpha^{*}\right\|_{2}^{2}=0$; that is,

$$
\left(A-A^{T}\right) \alpha^{*}=0 .
$$

Note that $b_{1}=\cdots=b_{r}=0, r \geq 1$, because 0 is the smallest eigenvalue of $-\left(A-A^{T}\right)^{2}$. Based on the definition of $\mathscr{J}_{2}$, we have

$$
a_{1}=\cdots=a_{r}, \quad \mathscr{J}_{2}=\{1, \ldots, r\}, \quad r \geq 1 .
$$

Applying Lemma 3 to (25), we know that $\alpha^{*}$ should be a linear combination of $u_{1}, \ldots, u_{r}$. Let

$$
\alpha^{*}=\sum_{j \in \mathscr{F}_{2}} \gamma_{j} u_{j}, \quad \gamma_{j} \in R .
$$

By (8), we have

$$
\left(A+A^{T}\right) u_{j}=2 a_{j} u_{j}=2 a_{1} u_{j}, \quad j \in \mathscr{J}_{2} .
$$

Therefore,

$$
\frac{A+A^{T}}{2} \alpha^{*}=\frac{1}{2}\left(\sum_{j \in \mathscr{F}_{2}} \gamma_{j}\left(A+A^{T}\right) u_{j}\right)=a_{1} \alpha^{*} .
$$

Then, by (26) and (30), it is straightforward to verify

$$
A \alpha^{*}=\frac{A+A^{T}}{2} \alpha^{*}+\frac{A-A^{T}}{2} \alpha^{*}=a_{1} \alpha^{*},
$$

thus proving the lemma.
In the case of $b_{1} \neq 0$, that is, all the eigenvalues of $-(A-$ $\left.A^{T}\right)^{2}$ are complex numbers, we have the following lemma similar to Lemma 7.

Lemma 10. Assume that (C2) holds. Given any nonzero $4 b_{1}^{2}$, the smallest eigenvalue of $-\left(A-A^{T}\right)^{2}$, and the corresponding eigenvector $\alpha^{*}$ obtained by (25), let $a_{1}=\left(\alpha^{*}\right)^{T}\left(A+A^{T}\right) \alpha^{*} /$ $2\left(\alpha^{*}\right)^{T} \alpha^{*}$ and $\beta^{*}=\left(A-A^{T}\right) \alpha^{*} / 2\left|b_{1}\right|$. Then, $a_{1} \pm i\left|b_{1}\right|$ are two eigenvalues of $A$ corresponding to the eigenvectors $\beta^{*} \pm i \alpha^{*}$, respectively.

Proof. The proof is almost the same to that in Lemma 7.

Remark 11. Among the following four real normal matrices

$$
\begin{aligned}
& \left(\begin{array}{ccccc}
7 & 0 & 0 & 0 & 0 \\
0 & 5 & 0 & 0 & 0 \\
0 & 0 & 5 & 0 & 0 \\
0 & 0 & 0 & 6 & 2 \\
0 & 0 & 0 & -2 & 6
\end{array}\right), \quad\left(\begin{array}{ccccc}
7 & 0 & 0 & 0 & 0 \\
0 & 5 & 1 & 0 & 0 \\
0 & -1 & 5 & 0 & 0 \\
0 & 0 & 0 & 6 & 2 \\
0 & 0 & 0 & -2 & 6
\end{array}\right), \\
& \left(\begin{array}{ccccc}
7 & 0 & 0 & 0 & 0 \\
0 & 5 & -2 & 0 & 0 \\
0 & 2 & 5 & 0 & 0 \\
0 & 0 & 0 & 5 & 2 \\
0 & 0 & 0 & -2 & 5
\end{array}\right), \quad\left(\begin{array}{ccccc}
7 & 0 & 0 & 0 & 0 \\
0 & 5 & 2 & 0 & 0 \\
0 & -2 & 5 & 0 & 0 \\
0 & 0 & 0 & 6 & 2 \\
0 & 0 & 0 & -2 & 6
\end{array}\right),
\end{aligned}
$$

only the first three meet (C1), but the last one does not. And only the last three matrices meet (C2), but the first one does not.

2.2. Computing the Eigenvalues with the Largest or Smallest Real Parts, as well as the Corresponding Eigenvectors. As shown in (8), $2 a_{j}, j=1, \ldots, r+k$, are the eigenvalues of the symmetric matrix $\left(A+A^{T}\right)$. In this subsection, we assume that $a_{1} \leq \cdots \leq a_{r}$ and $a_{r+1} \leq \cdots \leq a_{r+k}$, which can be achieved by reordering $a_{j}$ and the corresponding columns of $U$.

If replacing $A$ with $\left(A+A^{T}\right)$ in (2), we get

$$
\begin{aligned}
\frac{\mathrm{d} x(t)}{\mathrm{d} t}= & x(t)^{T} x(t)\left(A+A^{T}\right) x(t) \\
& -x(t)^{T}\left(A+A^{T}\right) x(t) x(t) .
\end{aligned}
$$

Without loss of generality, assume that $a_{r+k}$ is the largest real part of the eigenvalues of $A$ (it may be $a_{r}$. However, Lemmas 12 and 13 have no difference in that case). Applying Lemma 2 to (33), we can get $2 a_{r+k}=\lim _{t \rightarrow \infty}\left(x(t)^{T}(A+\right.$ $\left.\left.A^{T}\right) x(t) / x(t)^{T} x(t)\right)$, the largest eigenvalue of $\left(A+A^{T}\right)$, and the corresponding eigenvector $\zeta=\lim _{t \rightarrow \infty} x(t)$, where $x(t)$ is a solution of (33).

The following lemma introduces an approach for computing $\left|b_{r+k}\right|$ under the condition (C3).

Lemma 12. Assume that (C3) holds. Let $\zeta=\lim _{t \rightarrow \infty} x(t)$, where $x(t)$ is a solution of (33). Then, $\left|b_{r+k}\right|=$ $\sqrt{\left(\zeta^{T}\left(A A^{T}\right) \zeta / \zeta^{T} \zeta\right)-a_{r+k}^{2}}$ 
Proof. By Lemma 2, we know that $\zeta$ should be a linear combination of $u_{j}^{R}$ and $u_{j}^{I}, j \in \mathscr{J}_{3}$. Let

$$
\zeta=\sum_{j \in \mathscr{F}_{3}}\left(\gamma_{j} u_{j}^{R}+\tau_{j} u_{j}^{I}\right), \quad \gamma_{j}, \tau_{j} \in R .
$$

Then, based on (9), we get

$$
\begin{gathered}
\left(A A^{T}\right) u_{j}^{R}=\left(a_{j}^{2}+b_{j}^{2}\right) u_{j}^{R}, \\
\left(A A^{T}\right) u_{j}^{I}=\left(a_{j}^{2}+b_{j}^{2}\right) u_{j}^{I}, \quad j \in \mathscr{F}_{3} .
\end{gathered}
$$

Because $U$ is an orthogonal matrix and for all $j \in \mathscr{J}_{3}, a_{j}=$ $a_{r+k}$ and $\left|b_{j}\right|=\left|b_{r+k}\right|$ hold, that is, $a_{j}^{2}+b_{j}^{2}=a_{r+k}^{2}+b_{r+k}^{2}$ for all $j \in \mathscr{J}_{3}$, we have

$$
\frac{\zeta^{T}\left(A A^{T}\right) \zeta}{\zeta^{T} \zeta}=\frac{\left(a_{r+k}^{2}+b_{r+k}^{2}\right) \sum_{j \in \mathcal{F}_{3}}\left(\gamma_{j}^{2}+\tau_{j}^{2}\right)}{\sum_{j \in \mathcal{F}_{3}}\left(\gamma_{j}^{2}+\tau_{j}^{2}\right)}=a_{r+k}^{2}+b_{r+k}^{2},
$$

thus proving the lemma.

The following lemma introduces an approach for computing a pair of conjugated eigenvectors of $A$ corresponding to the eigenvalues $a_{r+k} \pm i\left|b_{r+k}\right|$ under the condition (C3).

Lemma 13. Assume that (C3) holds. Given any nonzero $2 a_{r+k}$, the largest eigenvalue of $\left(A+A^{T}\right)$, and the corresponding eigenvector $\zeta$ obtained by (33), let $\left|b_{r+k}\right|=$ $\sqrt{\left(\zeta^{T}\left(A A^{T}\right) \zeta / \zeta^{T} \zeta\right)-a_{r+k}^{2}}$. If $b_{r+k}=0, a_{r+k}$ is an eigenvalue of $A$ corresponding to the eigenvector $\zeta$. If $b_{r+k} \neq 0$, let $\eta=$ $\left(A-A^{T}\right) \zeta / 2\left|b_{r+k}\right|$. Then, $a_{r+k} \pm i\left|b_{r+k}\right|$ are two eigenvalues of $A$ corresponding to the eigenvectors $\eta \pm i \zeta$, respectively.

Proof. Combining the proofs of Lemmas 9 and 7, we can prove this lemma.

If replacing $A$ with $\left(A+A^{T}\right)$ in (3), we get

$$
\begin{aligned}
\frac{\mathrm{d} x(t)}{\mathrm{d} t}= & -x(t)^{T} x(t)\left(A+A^{T}\right) x(t) \\
& +x(t)^{T}\left(A+A^{T}\right) x(t) x(t) .
\end{aligned}
$$

Without loss of generality, assume that $a_{r+1}$ is the smallest real part of the eigenvalues of $A$ (it may be $a_{1}$. However, Lemma 14 has no difference in that case). Applying Lemma 3 to (37), we can obtain $2 a_{r+1}$, the smallest eigenvalue of $\left(A+A^{T}\right)$, as well as the corresponding eigenvector, denoted by $\zeta^{*}$. Then, we have the following lemma.

Lemma 14. Assume that (C4) holds. Given any nonzero $2 a_{r+1}$, the smallest eigenvalue of $\left(A+A^{T}\right)$, and the corresponding eigenvector $\zeta^{*}$ obtained by (37), let $\left|b_{r+1}\right|=$ $\sqrt{\left(\left(\zeta^{*}\right)^{T}\left(A A^{T}\right) \zeta^{*} /\left(\zeta^{*}\right)^{T} \zeta^{*}\right)-a_{r+1}^{2}}$. If $b_{r+1}=0, a_{r+1}$ is the eigenvalue of $A$ corresponding to the eigenvector $\zeta^{*}$. If $b_{r+1} \neq 0$, let $\eta^{*}=\left(A-A^{T}\right) \zeta^{*} / 2\left|b_{r+1}\right|$. Then, $a_{r+1} \pm i\left|b_{r+1}\right|$ are two eigenvalues of $A$ corresponding to the eigenvectors $\eta^{*} \pm i \zeta^{*}$, respectively.
Proof. Combining the proofs of Lemmas 12, 9, and 7, we can prove this lemma.

Remark 15. Among the following four real normal matrices

$$
\begin{aligned}
& \left(\begin{array}{ccccc}
1 & 0 & 0 & 0 & 0 \\
0 & 4 & 0 & 0 & 0 \\
0 & 0 & 5 & 0 & 0 \\
0 & 0 & 0 & 1 & 2 \\
0 & 0 & 0 & -2 & 1
\end{array}\right), \quad\left(\begin{array}{ccccc}
3 & 0 & 0 & 0 & 0 \\
0 & 4 & 0 & 0 & 0 \\
0 & 0 & 4 & 0 & 0 \\
0 & 0 & 0 & 1 & 2 \\
0 & 0 & 0 & -2 & 1
\end{array}\right), \\
& \left(\begin{array}{ccccc}
3 & 0 & 0 & 0 & 0 \\
0 & 4 & 2 & 0 & 0 \\
0 & -2 & 4 & 0 & 0 \\
0 & 0 & 0 & 4 & 2 \\
0 & 0 & 0 & -2 & 4
\end{array}\right), \quad\left(\begin{array}{ccccc}
3 & 0 & 0 & 0 & 0 \\
0 & 4 & 0 & 0 & 0 \\
0 & 0 & 5 & 0 & 0 \\
0 & 0 & 0 & 5 & 2 \\
0 & 0 & 0 & -2 & 5
\end{array}\right),
\end{aligned}
$$

only the first three meet (C3), but the last one does not. And only the last three matrices meet (C4), but the first one does not.

2.3. Computing the Eigenvalues with the Largest or Smallest Modulus, as well as the Corresponding Eigenvectors. Reorder the eigenvalues of the symmetric matrix $A A^{T}$ in (9) and the corresponding columns of $U$ such that $a_{1}^{2} \leq \cdots \leq a_{r}^{2}$ and $a_{r+1}^{2}+b_{r+1}^{2} \leq \cdots \leq a_{r+k}^{2}+b_{r+k}^{2}$. Without loss of generality, assume that $a_{r+k} \pm i\left|b_{r+k}\right|$ are the eigenvalues of $A$ that have the largest modulus (it may be $a_{r}$. However, Lemma 16 has no difference in that case), and that $a_{r+1} \pm i\left|b_{r+1}\right|$ are the eigenvalues of $A$ that have the smallest modulus (it may be $a_{1}$. However, Lemma 17 has no difference in that case).

Replacing $A$ with $A A^{T}$ in (2), we get

$$
\frac{\mathrm{d} x(t)}{\mathrm{d} t}=x(t)^{T} x(t) A A^{T} x(t)-x(t)^{T} A A^{T} x(t) x(t) .
$$

Applying Lemma 2 to (39), we can obtain $a_{r+k}^{2}+b_{r+k}^{2}=$ $\lim _{t \rightarrow \infty}\left(x(t)^{T} A^{T} A x(t) / x(t)^{T} x(t)\right)$, the largest eigenvalue of $A A^{T}$, and the corresponding eigenvector $\theta=\lim _{t \rightarrow \infty} x(t)$, where $x(t)$ is the solution of (39). Then, we have the following lemma.

Lemma 16. Assume that (C5) holds. Given any $a_{r+k}^{2}+b_{r+k}^{2}$, the largest eigenvalue of $A A^{T}$, and the corresponding eigenvector $\theta$ obtained by (39). Then, $a_{r+k}=\theta^{T}\left(A+A^{T}\right) \theta / 2 \theta^{T} \theta$. Thus, $\left|b_{r+k}\right|$ can be gotten. If $b_{r+k}=0, a_{r+k}$ is an eigenvalue of $A$ corresponding to the eigenvector $\theta$. If $b_{r+k} \neq 0$, let $\omega=(A-$ $\left.A^{T}\right) \theta /\left|2 b_{r+k}\right|$. Then, $a_{r+k} \pm i\left|b_{r+k}\right|$ are two eigenvalues of $A$ corresponding to the eigenvectors $\omega \pm i \theta$, respectively.

Proof. Combining the proofs of Lemmas 5, 7, and 9, we can prove this lemma.

Replacing $A$ with $A A^{T}$ in (3), we get

$$
\frac{\mathrm{d} x(t)}{\mathrm{d} t}=-x(t)^{T} x(t) A A^{T} x(t)+x(t)^{T} A A^{T} x(t) x(t) .
$$


Applying Lemma 3 to (40), we can obtain $a_{r+1}^{2}+b_{r+1}^{2}$, the smallest eigenvalue of $A A^{T}$, and the corresponding eigenvector, denoted by $\theta^{*}$. Then, we have the following lemma.

Lemma 17. Assume (C6) holds. Given any $a_{r+1}^{2}+b_{r+1}^{2}$, the smallest eigenvalue of $A A^{T}$, and the corresponding eigenvector $\theta^{*}$ obtained by (40). Then, $a_{r+1}=\left(\theta^{*}\right)^{T}\left(A+A^{T}\right) \theta^{*} / 2\left(\theta^{*}\right)^{T} \theta^{*}$. So one can get that $\left|b_{r+1}\right|$. If $b_{r+1}=0, a_{r+1}$ is an eigenvalue of $A$ corresponding to the eigenvector $\theta^{*}$. If $b_{r+1} \neq 0$, let $\omega^{*}=$ $\left(A-A^{T}\right) \theta^{*} / 2\left|b_{r+1}\right|$. Then, $a_{r+1} \pm i\left|b_{r+1}\right|$ are two eigenvalues of A corresponding to the eigenvectors $\omega^{*} \pm i \theta^{*}$, respectively.

Proof. Combining the proofs of Lemmas 5, 7, and 9, we can prove this lemma.

Remark 18. Among the following four real normal matrices

$$
\begin{array}{ll}
\left(\begin{array}{ccccc}
2 & 0 & 0 & 0 & 0 \\
0 & -2 & 0 & 0 & 0 \\
0 & 0 & 6 & 0 & 0 \\
0 & 0 & 0 & 1 & 2 \\
0 & 0 & 0 & -2 & 1
\end{array}\right), & \left(\begin{array}{ccccc}
3 & 0 & 0 & 0 & 0 \\
0 & 6 & 0 & 0 & 0 \\
0 & 0 & 6 & 0 & 0 \\
0 & 0 & 0 & 1 & 2 \\
0 & 0 & 0 & -2 & 1
\end{array}\right), \\
\left(\begin{array}{cccc}
3 & 0 & 0 & 0 \\
0 & 3 & -4 & 0 \\
0 & 4 & 3 & 0 \\
0 & 0 & 0 & 3 \\
0 & 0 & 0 & -4
\end{array}\right), & \left(\begin{array}{ccccc}
3 & 0 & 0 & 0 & 0 \\
0 & 4 & 0 & 0 & 0 \\
0 & 0 & 5 & 0 & 0 \\
0 & 0 & 0 & 3 & 4 \\
0 & 0 & 0 & -4 & 3
\end{array}\right),
\end{array}
$$

only the first three meet (C5), but the last one does not. And only the last three meet (C6), but the first one does not.

However, there exists some specially constructed $A$ that meet none of $(\mathrm{C} 1)$ to $(\mathrm{C} 6)$, for example, $A=$ $\operatorname{diag}\left[A_{1}, A_{2}, A_{3}, A_{4}\right]$, where

$$
\begin{aligned}
A_{1}=\left(\begin{array}{cc}
-4 & 1 \\
-1 & -4
\end{array}\right), \quad A_{2} & =\left(\begin{array}{cc}
-4 & 3 \\
-3 & -4
\end{array}\right), \quad A_{3}=\left(\begin{array}{cc}
4 & 3 \\
-3 & 4
\end{array}\right), \\
A_{4} & =\left(\begin{array}{cc}
4 & 1 \\
-1 & 4
\end{array}\right) .
\end{aligned}
$$

Nevertheless, a randomly generated real normal matrix can meet (C1) to (C6) with high probability.

2.4. Extension to Arbitrary Real Matrices. In this subsection, $A$ is an arbitrary real matrix of order $n$. Let $\|A\|$ be the Frobenius norm of $A$, and $\lambda_{j}, j=1, \ldots, n$, be the eigenvalue of $A$. Denote the set of all complex nonsingular matrices by $\mathscr{T}$.

By the Schur inequality [13], we know

$$
\sum_{j=1}^{n}\left|\lambda_{j}^{2}\right| \leq\|A\|^{2}
$$

with equality if and only if $A$ is a normal matrix. Since the spectrum of $A$ does not change by a similarity transformation, the inequality

$$
\sum_{j=1}^{n}\left|\lambda_{j}^{2}\right| \leq\left\|P^{-1} A P\right\|^{2}, \quad P \in \mathscr{T}
$$

holds with equality if and only if $P^{-1} A P$ is a normal matrix. In addition, [24] proved

$$
\sum_{i=1}^{n}\left|\lambda_{i}^{2}\right|=\inf _{P \in \mathscr{T}}\left\|P^{-1} A P\right\|^{2} .
$$

Based on (45), if we can find a sequence $A_{j}$ as follows:

$$
\begin{array}{r}
A_{1}=A, \quad A_{j+1}=P_{j}^{-1} A_{j} P_{j}, \quad\left\|A_{j+1}\right\|<\left\|A_{j}\right\|, \\
P_{j} \in \mathscr{T}, j=1,2, \ldots
\end{array}
$$

such that

$$
\sum_{i=1}^{n}\left|\lambda_{i}^{2}\right|=\lim _{j \rightarrow \infty}\left\|A_{j}\right\|^{2},
$$

where $A_{\infty}=\lim _{j \rightarrow \infty} A_{j}$ is to be a normal matrix with the same eigenvalues as $A$. Such skill, termed as the normreducing technique, has been proposed by [25-27]. Moreover, following the idea presented by [26], it is easy to find that when $A$ is real, $A_{\infty}$ can be chosen to be a real normal matrix.

In a word, any real matrix $A$ can be translated into a real normal matrix $A_{\infty}=P_{\infty}^{-1} A P_{\infty}$ by a similarity transformation $P_{\infty}=P_{1} P_{2} \cdots$. Typical approaches for constructing $P_{j}$ can be found in [26]. Note that if $\lambda$ is the eigenvalue of $A_{\infty}$ corresponding to the eigenvector $u, \lambda$ is the eigenvalue of $A$ corresponding to the eigenvector $P_{\infty} u$. Hence, our proposed algorithm can be extended to extract eigenpairs of arbitrary real matrices by employing the norm-reducing technique.

Without loss of generality, we use the following random matrix $A$ as an example to describe the norm-reducing technique:

$$
A=\left(\begin{array}{ccccc}
1.0541 & 0.4052 & -1.0191 & -0.5771 & 0.4158 \\
-1.9797 & -0.7025 & -1.3852 & -0.8364 & 0.0430 \\
-1.8674 & 1.4990 & 0.9549 & 0.8530 & -0.9489 \\
-1.8324 & 0.1378 & -0.6011 & 0.4773 & 0.5416 \\
0.8486 & -1.5868 & -1.1719 & 0.3023 & -0.8211
\end{array}\right)
$$

The Frobenius norm of $A$ is $\|A\|=\sqrt{27.7629}$, and the eigenvalues of matrix $A$ are $\lambda_{1}=2.8797, \lambda_{2}=\overline{\lambda_{3}}=$ $-0.6900+1.8770 i, \lambda_{4}=-1.5144$, and $\lambda_{5}=0.9774$; obviously $\|A\|>\sqrt{\sum_{i=1}^{5} \lambda_{i}^{2}}=19.5401$, so $A$ is a nonnormal real matrix. According to the approach that presented in [26], we can construct $P_{j}, j=1,2, \ldots$. If the condition $\left\|A_{i}-A_{i+1}\right\|<10^{-10}$ can be satisfied, we break the iteration. After 129 iterations, we can obtain a approximate normal matrix $A_{130}$ :

$$
A_{130}=\left(\begin{array}{ccccc}
-0.7441 & 0.0007 & 1.6334 & 0.8210 & 0.0010 \\
-0.0035 & 2.8797 & -0.0038 & 0.0000 & -0.0000 \\
-1.4162 & -0.0048 & -0.9132 & 0.8223 & 0.0034 \\
-1.1560 & -0.0019 & -0.1181 & -1.2370 & -0.0014 \\
0.0011 & -0.0000 & 0 & -0.0036 & 0.9774
\end{array}\right) \text {, }
$$

from which we have $\left\|A_{130}^{*} A_{130}-A_{130} A_{130}^{*}\right\|=$ $\sqrt{0.00121231723027107}$ and $\left\|A_{130}\right\|=\sqrt{19.540133042214}$ 


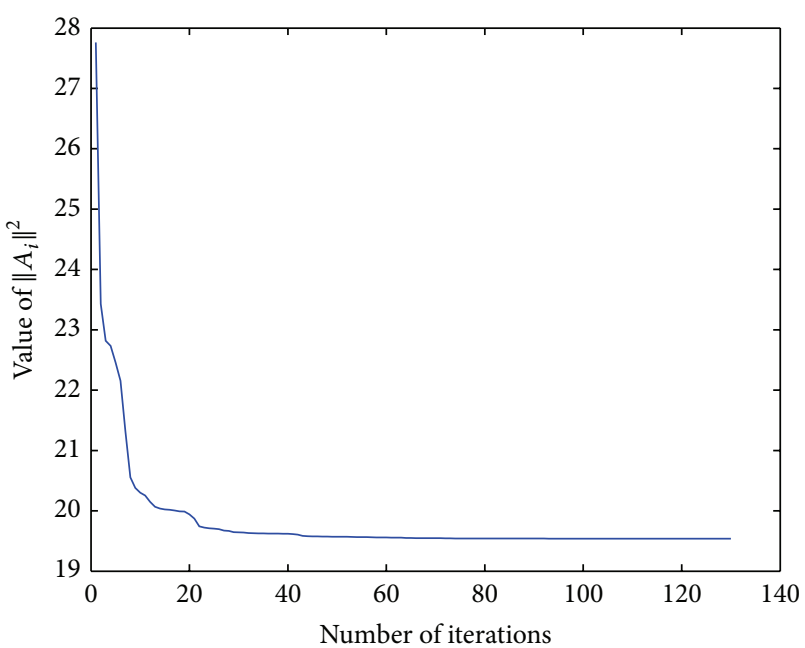

Figure 1: Trajectory of $\left\|A_{i}\right\|^{2}$.

that is very close to $\sqrt{\sum_{i=1}^{5} \lambda_{i}^{2}}=\sqrt{19.5400526824864}$; so the matrix $A_{130}$ can be regarded as a normal matrix in practical application, the corresponding $P_{\infty}=P_{1} P_{2} P_{3} \cdots P_{129}$ are as follows:

$P_{\infty}=\left(\begin{array}{ccccc}-0.3499 & -0.5290 & -0.0090 & 0.2228 & -0.0151 \\ -0.4809 & -0.0259 & -0.6935 & -0.4079 & 0.1559 \\ -0.2914 & 0.7211 & 0.6743 & 0.0395 & 0.3437 \\ -0.4484 & 0.1475 & -0.3668 & 0.2444 & -0.8704 \\ 0.0611 & -0.3265 & 0.4294 & -1.1028 & -0.5166\end{array}\right)$,

which satisfy $A_{130}=P_{\infty}^{-1} A P_{\infty}$. We also can see that the eigenvalues of $A_{130}$ are $\lambda_{1}=-1.5144, \lambda_{2}=\overline{\lambda_{3}}=-0.6900+$ $1.8770 i, \lambda_{4}=2.8797$, and $\lambda_{5}=0.9774$, which are just the eigenvalues of original nonnormal matrix $A$. We presented here the transient behavior of $\left\|A_{i}\right\|^{2}$ in Figure 1, where $i=$ $1,2,3, \ldots$ is the iterations and $A_{1}=A$.

In the following, we use $N=1000$ random matrices to verify the average performance of the norm-reducing technique. Let

$$
\text { Mean } \Delta_{i}^{2}=\frac{1}{N} \sum_{j=1}^{N} \Delta_{j i}^{2}
$$

denotes the average measure of a large number of nonnormal matrices in a statistical sense at ith iteration, where $\Delta_{j i}^{2}=$ $\left\|A_{j i}^{T} A_{j i}-A_{j i} A_{j i}^{T}\right\|^{2}$ is the measure of the nonnormal matrix $A_{j}$ at $i$ th iteration, $\Delta_{j i}^{2}=0$ if and only if $A_{j}$ is normal matrix at $i$ th iteration. We also presented the dynamic behavior trajectory of Mean $\Delta^{2}$ in Figure 2, from which we can see that for most of nonnormal matrices, after 350 iterations, the Mean $\Delta^{2}$ is very close to zero.

\section{Neural Implementation Description}

In the presented paper, we mainly focus on the classical neural network differential equation as shown in (2), where

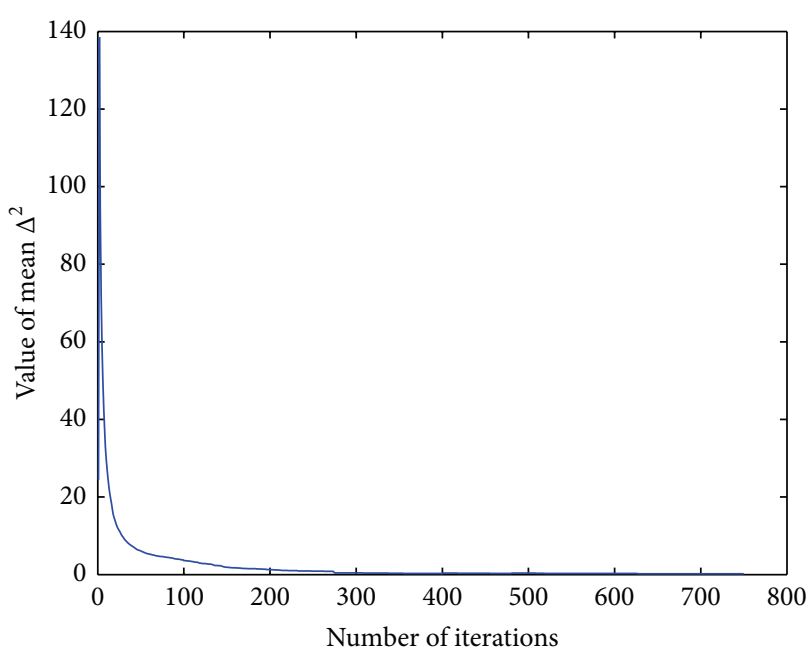

FIGURE 2: Average performance trajectory of norm-reducing technique.

$A=\left(a_{i j}\right), i, j=1,2, \ldots, n$ are symmetric matrices that need to calculate eigenvalues and the corresponding eigenvectors, $x(t)=\left[x_{1}(t), x_{2}(t), \ldots, x_{n}(t)\right]^{T}$ is a column vector which denotes the states of neurons in the neural network dynamic system, and the elements of symmetric matrix $A$ denote the connection weights between those neurons. We presented the schematic diagram of the neural network in Figure 3, from which we can see that it is a recurrent neural network since the input is just the output of the system.

In the practical applications, we often only need a nonzero column vector $x(0)=\left[x_{1}(0), x_{2}(0), \ldots, x_{n}(0)\right]^{T}$ to start the neural network system by the following update rule:

$$
\begin{aligned}
x(j+1)=x(j)+\tau( & \left(j(j)^{T} x(j) A x(j)\right. \\
& \left.-x(j)^{T} A x(j) x(j)\right),
\end{aligned}
$$

where $j$ denote the $j$ th iteration and $\tau$ is a small time step. The iteration stops once $\|x(j+1)-x(j)\|<\varepsilon$, where $\varepsilon$ is a small constraint error that can be set in advance. If $\| x(j+1)-$ $x(j) \|<\varepsilon$, we could regard that $\|x(j+1)-x(j)\|=0$, that is, $x(j)^{T} x(j) A x(j)-x(j)^{T} A x(j) x(j)=0$; so we have $A x(j)=$ $\left(x(j)^{T} A x(j) / x(j)^{T} x(j)\right) x(j)$, according to the theory in [23], $x(j)$ is the eigenvector corresponding to the modulus largest eigenvalue which can be denoted as $x(j)^{T} A x(j) / x(j)^{T} x(j)$.

\section{Examples and Discussion}

Three experiments are presented to verify our results. The following real normal matrix $A$ (randomly generated) was used in those three experiments:

$$
A=\left(\begin{array}{cccccc}
1.8818 & -2.1563 & -0.5979 & -0.4768 & 0.7270 & 0.4133 \\
-1.4267 & 0.5258 & 1.4946 & 0.7125 & 0.4700 & 2.5384 \\
0.4273 & 0.7784 & 2.8645 & 0.8587 & 1.3792 & -1.0153 \\
-0.1929 & 1.3273 & -1.3125 & 3.1629 & 0.2573 & 0.6329 \\
1.5966 & -0.6593 & -0.3799 & 0.2058 & 2.9845 & 0.6404 \\
1.0627 & 1.9973 & -0.0249 & -1.5727 & 0.9289 & 2.3259
\end{array}\right)
$$




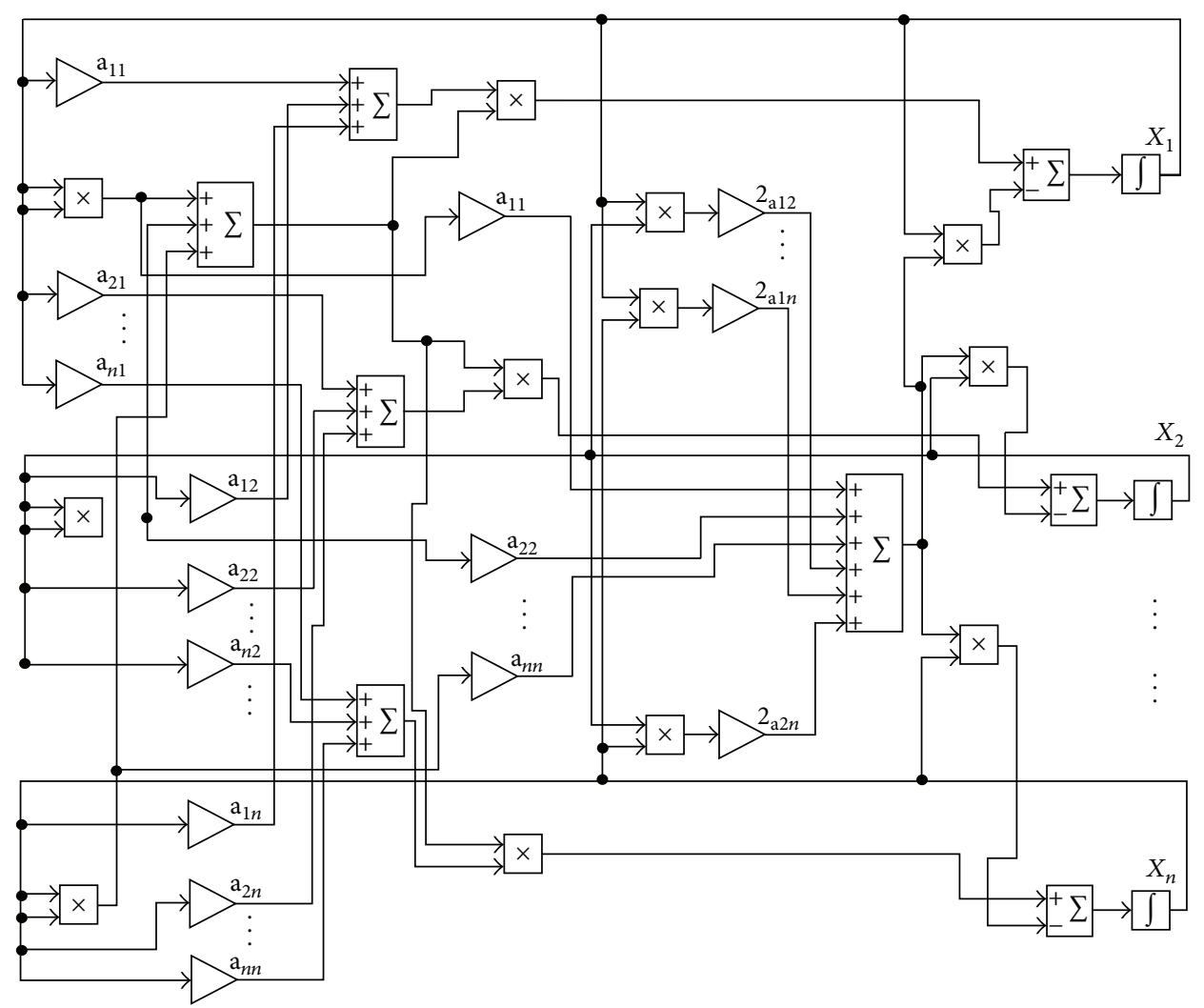

FIGURE 3: The schematic diagram of the neural network equation (2) for solving the eigenvector corresponding to the modulus largest eigenvalues of real symmetric matrix $A$.

Using the $[V, D]=\operatorname{eig}(A)$ function in Matlab, we got the eigenvalues of $A$ as $\lambda_{1}=-2.3420, \lambda_{2}=1.005, \lambda_{3}=\overline{\lambda_{4}}=$ $3.1967+1.7860 i$, and $\lambda_{5}=\overline{\lambda_{6}}=4.3445+1.2532 i$, as well as the corresponding eigenvectors $u_{1}, \ldots, u_{6}$ as follows:

$$
\begin{gathered}
u_{1}=\left(\begin{array}{c}
0.3754 \\
0.7510 \\
-0.2146 \\
-0.1664 \\
0.0280 \\
-0.4696
\end{array}\right), \\
u_{2}=\left(\begin{array}{c}
0.6657 \\
-0.0242 \\
0.2994 \\
0.2594 \\
-0.5888 \\
0.2295
\end{array}\right), \\
u_{3}=\left(\begin{array}{c}
-0.0294+0.1297 i \\
-0.0243-0.1385 i \\
0.6165 \\
-0.1917+0.5472 i \\
0.0913+0.2785 i \\
-0.2708-0.2950 i
\end{array}\right),
\end{gathered}
$$

$$
u_{5}=\left(\begin{array}{c}
0.3207+0.2956 i \\
0.0472-0.4424 i \\
0.0711-0.2167 i \\
-0.1146-0.3213 i \\
0.4903 \\
0.3692-0.2583 i
\end{array}\right),
$$

We can see that all of (C1) to (C6) hold except (C2). For simplicity, denote $\lim _{t \rightarrow \infty} x(t)$ by $x(\infty)$.

Example 19 (for Section 2.1). We used (10) with the following initial condition (randomly generalized)

$$
\begin{array}{r}
x(0)=[0.2329,-0.0023,-0.8601, \\
\quad-0.2452,-0.1761,-0.1577]^{T}
\end{array}
$$

to get $\left|b_{3}\right|$ (the largest absolute value of the imaginary part among $\left.\lambda_{i}\right)$ that $\sqrt{-x(t)^{T}\left(A-A^{T}\right)^{2} x(t) / 4 x(t)^{T} x(t)}$ should converge to. From Lemma $5, \quad x(t)^{T}(A+$ $\left.A^{T}\right) x(t) / 2 x(t)^{T} x(t) \rightarrow a_{3}$ should hold. By Lemma 7, $x(t)$ and $\left(A-A^{T}\right) x(t) / 2\left|b_{3}\right|$ should converge to the imaginary 


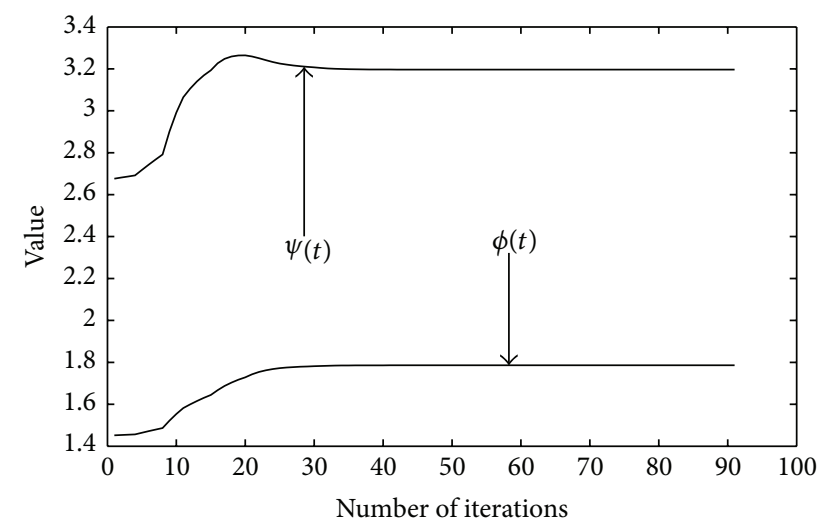

FIgURE 4: Trajectories of $\phi(t)=\sqrt{-x(t)^{T}\left(A-A^{T}\right)^{2} x(t) / 4 x(t)^{T} x(t)}$ and $\psi(t)=x(t)^{T}\left(A+A^{T}\right) x(t) / 2 x(t)^{T} x(t)$ based on (10) with initial $x(0)$ as (55), which should converge to $\left|b_{3}\right|$ and $a_{3}$, respectively.

and real parts of an eigenvector corresponding to $\lambda_{3}$, respectively. The transient behaviors of the above four variables are shown in Figures 4, 5, and 6, respectively. After convergence, we saw

$$
\begin{aligned}
& \frac{\left(A-A^{T}\right) x(\infty)}{2\left|b_{3}\right|}+x(\infty) i \\
& =\left(\begin{array}{c}
0.1795-0.0005 i \\
-0.1747+0.0737 i \\
-0.1857-0.8107 i \\
0.7772+0.0872 i \\
0.3386-0.2039 i \\
-0.3064+0.4450 i
\end{array}\right) \\
& =(-0.3012-1.3149 i) u_{3} .
\end{aligned}
$$

Thus, the estimated complex vector is an eigenvector of $A$ corresponding to $\lambda_{3}$.

Although we can use (25) to get the smallest absolute value of the imaginary part among $\lambda_{i}$ (it is zero in this experiment), neither the corresponding real part nor the eigenvector can be obtained from Lemmas 8 or 9 since (C2) does not hold.

Example 20 (for Section 2.2). We used (33) with the same $x(0)$ as (55) to get $a_{5}$ (the largest real part among $\lambda_{i}$ ) that $x(t)^{T}\left(A+A^{T}\right) x(t) / 2 x(t)^{T} x(t)$ should converge to. Based on Lemma 12, $\sqrt{\left(x(t)^{T}\left(A A^{T}\right) x(t) / x(t)^{T} x(t)\right)-a_{5}^{2}} \rightarrow\left|b_{5}\right|$ should hold. The transient behaviors of such two variables are shown in Figure 7. Since $b_{5} \neq 0$, from Lemma $13, x(t)$ and $\left(A-A^{T}\right) x(t) / 2\left|b_{5}\right|$ should converge to the imaginary and real parts of an eigenvector corresponding to $\lambda_{5}$, as shown

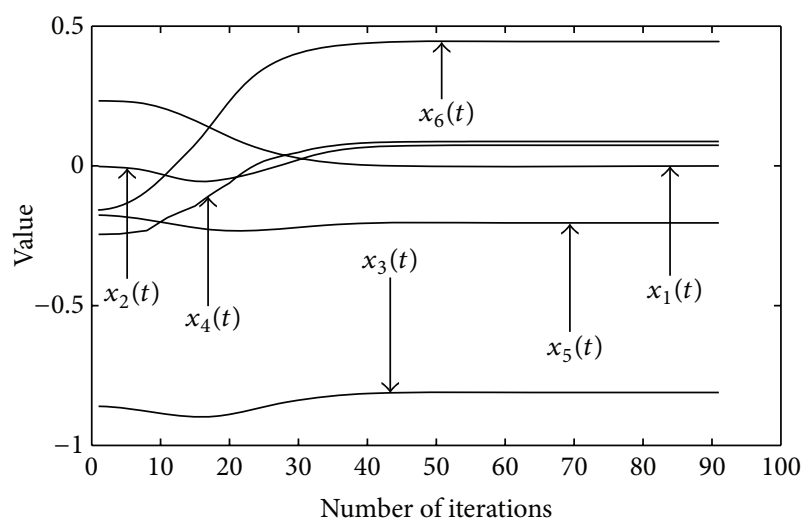

FIgURE 5: Trajectories of $x(t)$, the solution of (10) with initial $x(0)$ as (55), which should converge to the imaginary part of an eigenvector of $A$ corresponding to the eigenvalue $\lambda_{3}$.

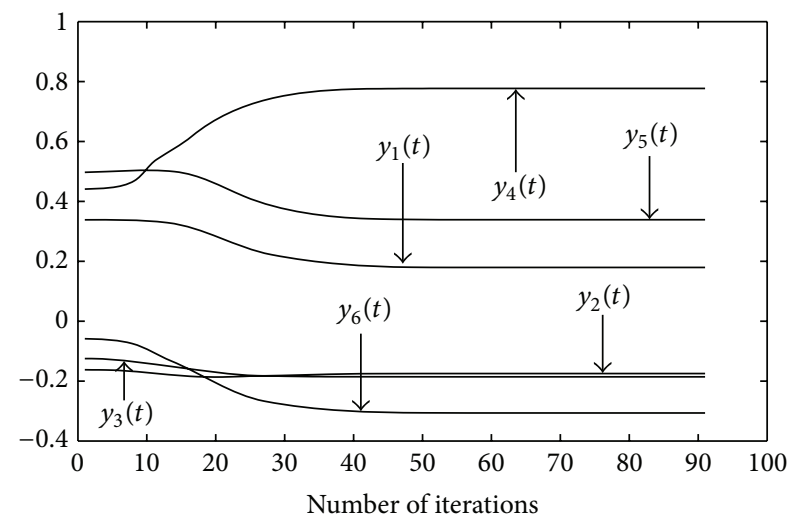

Figure 6: Trajectories of $y(t)=\left(A-A^{T}\right) x(t) / 2 b_{3}$ based on (10) with initial $x(0)$ as (55), which should converge to the real part of an eigenvector of $A$ corresponding to the eigenvalue $\lambda_{3}$.

in Figures 8 and 9. After convergence, we saw

$$
\begin{aligned}
& \frac{\left(A-A^{T}\right) x(\infty)}{2\left|b_{5}\right|}+x(\infty) i \\
& =\left(\begin{array}{c}
-0.5695-0.1474 i \\
0.2254+0.5561 i \\
0.0534+0.3029 i \\
0.3407+0.3091 i \\
-0.5830+0.3120 i \\
-0.2746+0.5420 i
\end{array}\right) \\
& =(-1.1890+0.6363 i) u_{5} .
\end{aligned}
$$

Hence, the estimated complex vector is an eigenvector of $A$ corresponding to $\lambda_{5}$.

Based on (37), we got $a_{1}$ (the smallest real part among $\left.\lambda_{i}\right)$. After convergence, we saw that $x(\infty)^{T}(A+$ $\left.A^{T}\right) x(\infty) / 2 x(\infty)^{T} x(\infty)=a_{1}=\lambda_{1}=-2.3420$, $\sqrt{\left(x(\infty)^{T}\left(A A^{T}\right) x(\infty) / x(\infty)^{T} x(\infty)\right)-a_{1}^{2}}=\left|b_{1}\right|=0$, and 




Figure 7: Trajectories of $\psi(t)=x(t)^{T}\left(A+A^{T}\right) x(t) / 2 x(t)^{T} x(t)$ and $\phi(t)=\sqrt{\left(x(t)^{T}\left(A A^{T}\right) x(t) / x(t)^{T} x(t)\right)-a_{5}^{2}}$ based on (33) with initial $x(0)$ as (55), which should converge to $a_{5}$ and $\left|b_{5}\right|$, respectively.

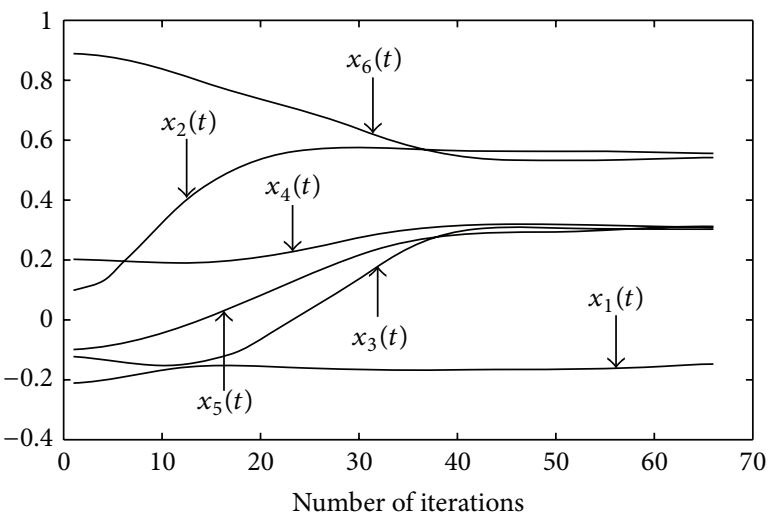

FIGURE 8: Trajectories of $x(t)$, the solution of (33) with initial $x(0)$ as (55), which should converge to the imaginary part of an eigenvector of $A$ corresponding to the eigenvalue $\lambda_{5}$.

$x(\infty)$ was equal to

$$
x(\infty)=\left(\begin{array}{c}
-0.3581 \\
-0.7165 \\
0.2048 \\
0.1588 \\
-0.0267 \\
0.4480
\end{array}\right)=-0.9541 u_{1}
$$

just as expected from Lemma 14.

Example 21 (for Section 2.3). Based on (39) and Lemma 16, we can get $\lambda_{5}$ and one corresponding eigenvector again because $\left|\lambda_{5}\right|$ is the largest modulus of $\lambda_{i}$. In addition, we used (40) with the same $x(0)$ as (55) to get $\left|\lambda_{2}\right|$, the smallest modulus of $\lambda_{i}$. By Lemma 17, $a_{2}=x(\infty)^{T}\left(A+A^{T}\right) x(\infty) / 2 x(\infty)^{T} x(\infty)$ and $\left|b_{2}\right|=\sqrt{\left(x(\infty)^{T}\left(A A^{T}\right) x(\infty) / x(\infty)^{T} x(\infty)\right)-a_{2}^{2}}$ should hold. The transient behaviors of such two variables are shown in Figure 10. After convergence, we saw that $b_{2}=0$. Hence, from Lemma 17, $x(t)$ should converge to a constant multiple

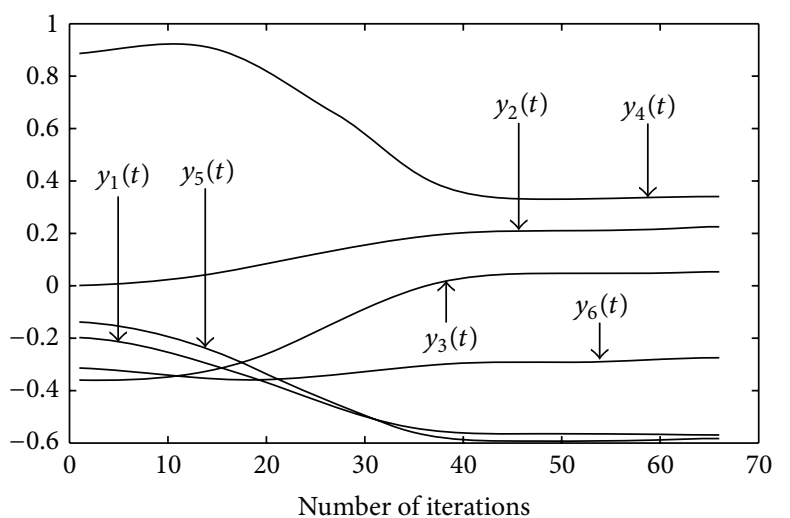

Figure 9: Trajectories of $y(t)=\left(A-A^{T}\right) x(t) / 2\left|b_{5}\right|$ based on (33) with initial $x(0)$ as (55), which should converge to the real part of an eigenvector of $A$ corresponding to the eigenvalue $\lambda_{5}$.

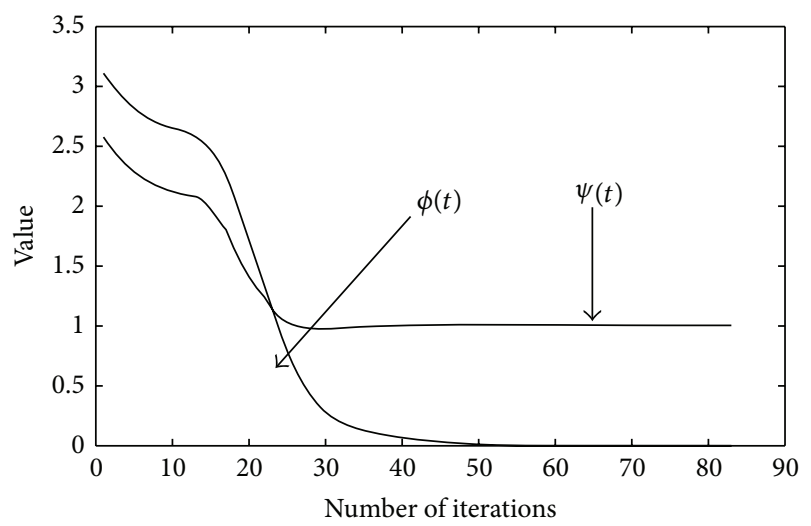

FIgURE 10: Trajectories of $\psi(t)=x(t)^{T}\left(A+A^{T}\right) x(t) / 2 x(t)^{T} x(t)$ and $\phi(t)=\sqrt{\left(x(t)^{T}\left(A A^{T}\right) x(t) / x(t)^{T} x(t)\right)-a_{2}^{2}}$ based on (40) with initial $x(0)$ as (55), which should converge to $a_{2}$ and $\left|b_{2}\right|$, respectively.

of $u_{2}$, which is shown in Figure 11. As expected, $x(\infty)$ was equal to

$$
x(\infty)=\left(\begin{array}{c}
-0.6337 \\
0.0230 \\
-0.2850 \\
-0.2469 \\
0.5605 \\
-0.2185
\end{array}\right)=-0.9519 u_{2}
$$

Example 22 (for extension to arbitrary real matrices). According to the theory in Section 2.1, we present here an experiment for arbitrary real matrices to verify the effectiveness of the norm-reducing technique in Section 2.4. Considering the following nonnormal matrix $A$ :

$$
A=\left(\begin{array}{ccccc}
3.4633 & -3.7487 & 2.9281 & -0.5106 & -0.0455 \\
4.4860 & 3.4259 & -3.2010 & 5.0625 & 1.2120 \\
0.5268 & -1.0311 & 1.5386 & -0.4766 & -1.4478 \\
-2.4768 & 1.2585 & 2.0525 & 2.3539 & -0.8487 \\
0.0498 & -3.1267 & -6.5297 & -1.5106 & 2.2083
\end{array}\right) \text {, }
$$




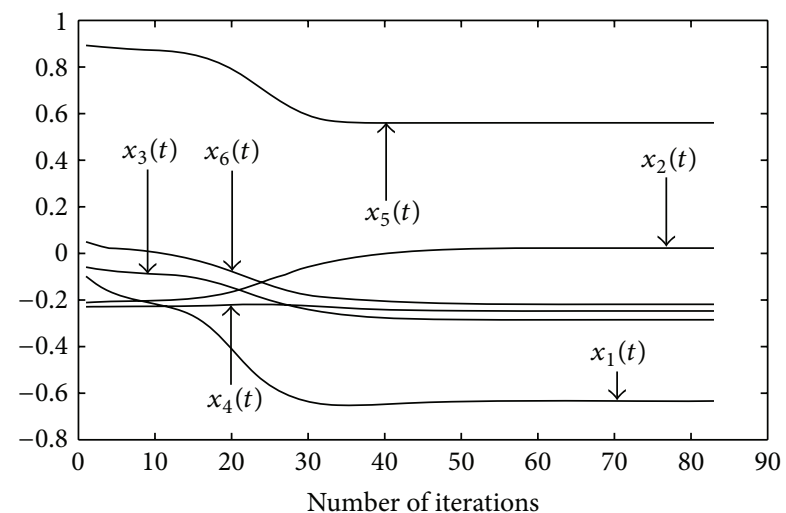

FIgURE 11: Trajectories of $x(t)$, the solution of (40), with initial $x(0)$ as (55), which should converge to an eigenvector of $A$ corresponding to the eigenvalue $\lambda_{2}$.



FIgURE 12: Trajectories of $\phi(t)=$ $\sqrt{-x(t)^{T}\left(A_{p}-A_{p}^{T}\right)^{2} x(t) / 4 x(t)^{T} x(t)}$ and $\psi(t)=x(t)^{T}\left(A_{p}+\right.$ $\left.A_{p}^{T}\right) x(t) / 2 x(t)^{T} x(t)$ based on (10) with initial $x(0)$ as (65), which should converge to $\left|b_{2}\right|$ and $a_{2}$, respectively.

the Frobenius norm of $A$ is $\|A\|=\sqrt{187.8869}>\sqrt{\sum_{i=1}^{5} \lambda_{i}^{2}}=$ 97.1780, where $\lambda_{i}, i=1,2, \ldots, 5$ are the eigenvalues of matrix $A$, so matrix $A$ is a nonnormal matrix. According to the theory in Section 2.4, the eigenpairs problem of nonnormal real matrix can be converted into the eigenpairs problem of the corresponding normal matrix $A_{p}$, which can be calculated by norm-reducing technique:

$$
A_{p}=\left(\begin{array}{ccccc}
2.3438 & 0.7915 & 1.1071 & 1.8624 & -2.6867 \\
-1.5918 & 2.1016 & 1.7249 & 2.2067 & 1.9843 \\
0.8659 & -1.7434 & 4.5103 & -1.2708 & 0.0408 \\
1.7777 & -2.3015 & -0.9898 & 3.5815 & 0.7201 \\
-2.4688 & -2.3136 & -0.4746 & 0.0545 & 0.4528
\end{array}\right)
$$

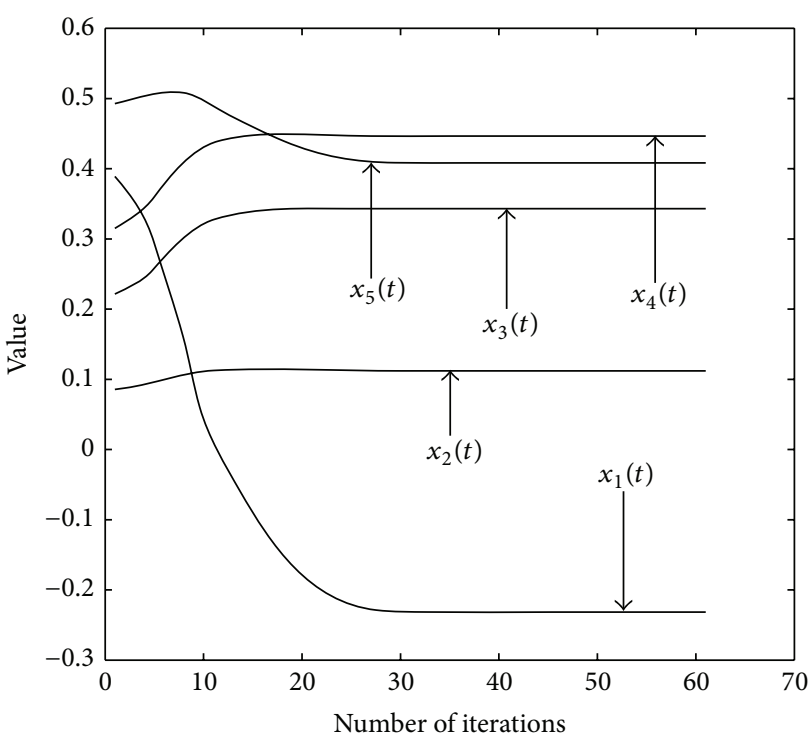

FIGURE 13: Trajectories of $x(t)$, the solution of (10) with initial $x(0)$ as (65), which should converge to the imaginary part of an eigenvector of $A_{p}$ corresponding to the eigenvalue $\lambda_{2}$.

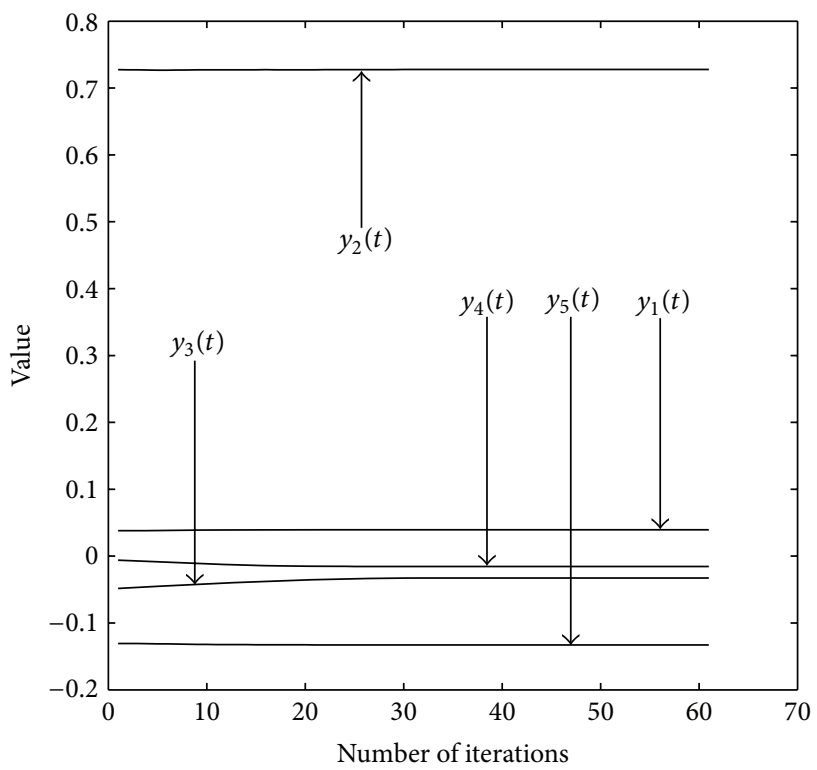

FIGURE 14: Trajectories of $y(t)=\left(A_{p}-A_{p}^{T}\right) x(t) / 2 b_{2}$ based on (10) with initial $x(0)$ as (65), which should converge to the real part of an eigenvector of $A_{p}$ corresponding to the eigenvalue $\lambda_{2}$.

and the corresponding $P_{\infty}$ as follows:

$$
P_{\infty}=\left(\begin{array}{ccccc}
28.2974 & -28.8442 & 115.6698 & 16.4765 & 13.5575 \\
-35.9689 & 48.5475 & 4.3465 & 58.1384 & 9.0642 \\
-10.2373 & -14.2976 & 14.1168 & 23.6416 & -40.2882 \\
-3.4360 & -25.4122 & -114.2417 & 15.0191 & 15.1720 \\
-79.1532 & -35.3465 & 75.4347 & -24.8455 & -48.2893
\end{array}\right) \text {, }
$$

which satisfies the relationship $A_{p}=P_{\infty}^{-1} A P_{\infty}$. In order to solve the eigenpairs of matrix $A$, we have to calculate the eigenpairs of the matrix $A_{p}$ at first. Direct calculations 
of the eigenvalues of matrix $A_{p}$ are $\lambda_{1}=-1.8342, \lambda_{2}=$ $\overline{\lambda_{3}}=2.1114+3.786 i, \lambda_{4}=\overline{\lambda_{5}}=5.3007+0.1328 i$ and those are just the eigenvalues of nonnormal matrix $A$ and the corresponding eigenvectors as follows:

$$
\begin{gathered}
u_{1}=\left(\begin{array}{c}
0.6079 \\
0.0886 \\
-0.1195 \\
-0.2805 \\
0.7278
\end{array}\right), \quad u_{2}=\left(\begin{array}{c}
0.0035-0.2240 i \\
0.7020 \\
0.0189+0.3280 i \\
0.0502+0.4231 i \\
-0.0660+0.4040 i
\end{array}\right)=\overline{u_{3}}, \\
u_{4}=\left(\begin{array}{c}
0.1769-0.4835 i \\
-0.0129+0.0561 i \\
0.6204 \\
-0.2522-0.4643 i \\
-0.1415+0.2181 i
\end{array}\right)=\overline{u_{5}} .
\end{gathered}
$$

The eigenvectors of nonnormal matrix $A$, which corresponding to the eigenvalues with largest imaginary parts in absolute are

$$
u_{2}^{A}=\left(\begin{array}{c}
-0.2353-0.5331 i \\
-0.6416 \\
0.0449-0.0581 i \\
0.3856+0.0270 i \\
0.0747-0.2973 i
\end{array}\right)=\overline{u_{3}^{A}} .
$$

According to the results above, the eigenvalues with largest imaginary in absolute are $\lambda_{2}$ and $\lambda_{3}$, and let $a_{2} \pm\left|b_{2}\right| i$ denote them.

We used (10) with the following initial condition (randomly generalized):

$$
x(0)=[0.3891,0.0856,0.2215,0.3151,0.4929]^{T}
$$

to get $\left|b_{2}\right|$ (the largest absolute value of the imaginary part among $\left.\lambda_{i}\right)$ that $\sqrt{-x(t)^{T}\left(A_{p}-A_{p}^{T}\right)^{2} x(t) / 4 x(t)^{T} x(t)}$ should converge to. From Lemma 5, $x(t)^{T}\left(A_{p}+\right.$ $\left.A_{p}^{T}\right) x(t) / 2 x(t)^{T} x(t) \rightarrow a_{2}$ should hold. By Lemma 7, $x(t)$ and $\left(A_{p}-A_{p}^{T}\right) x(t) / 2\left|b_{2}\right|$ should converge to the imaginary and real parts of an eigenvector corresponding to $\lambda_{2}$, respectively. The transient behaviors of the above four variables are shown in Figures 12, 13, and 14, respectively.

After convergence, we saw

$$
\begin{aligned}
& \frac{\left(A_{p}-A_{p}^{T}\right) x(\infty)}{2\left|b_{2}\right|}+x(\infty) i \\
& =\left(\begin{array}{c}
0.0394-0.2317 i \\
0.7277+0.1121 i \\
-0.0327+0.3430 i \\
-0.0155+0.4466 i \\
-0.1329+0.4083 i
\end{array}\right) \\
& =(1.0367+0.1597 \mathrm{i}) u_{2} .
\end{aligned}
$$

Thus, the estimated complex vector is an eigenvector of $A_{p}$ corresponding to $\lambda_{2}$. According to the theory in Section 2.4, the estimated complex eigenvector of nonnormal matrix $A$ corresponding to the eigenvalue $\lambda_{2}$ should be

$$
\begin{array}{r}
P_{\infty}\left(\frac{\left(A_{p}-A_{p}^{T}\right) x(\infty)}{2\left|b_{2}\right|}+x(\infty) i\right) \\
=\left(\begin{array}{c}
-25.7189+42.7862 i \\
31.6606+44.9313 i \\
-6.2833-0.2807 i \\
-17.1372-28.3402 i \\
-24.5113+9.4386 i
\end{array}\right) \\
=(-49.3476-70.0318 i) u_{2}^{A},
\end{array}
$$

from which we can see that the estimated complex vector is an eigenvector of $A$ corresponding to $\lambda_{2}$.

\section{Conclusion}

This paper introduces a neural network based approach for computing eigenvectors of real normal matrices and the corresponding eigenvalues that have the largest or smallest modulus, have the largest or smallest real part, and have the largest or smallest imaginary part in absolute value. All the computation can be carried out in real vector space although eigenpairs may be complex, which can reduce the scale of networks a lot. We also shed light on extending this method to the case of general real matrices by employing the norm-reducing technique proposed in other literatures. Four simulation examples verified the validity of our proposed algorithm.

\section{References}

[1] S. Attallah and K. Abed-Meraim, "A fast adaptive algorithm for the generalized symmetric eigenvalue problem," IEEE Signal Processing Letters, vol. 15, pp. 797-800, 2008.

[2] T. Laudadio, N. Mastronardi, and M. Van Barel, "Computing a lower bound of the smallest eigenvalue of a symmetric positivedefinite Toeplitz matrix," IEEE Transactions on Information Theory, vol. 54, no. 10, pp. 4726-4731, 2008.

[3] J. Shawe-Taylor, C. K. I. Williams, N. Cristianini, and J. Kandola, "On the eigenspectrum of the Gram matrix and the generalization error of kernel-PCA," IEEE Transactions on Information Theory, vol. 51, no. 7, pp. 2510-2522, 2005.

[4] D. Q. Wang and M. Zhang, "A new approach to multiple class pattern classification with random matrices," Journal of Applied Mathematics and Decision Sciences, no. 3, pp. 165-175, 2005.

[5] M. R. Bastian, J. H. Gunther, and T. K. Moon, "A simplified natural gradient learning algorithm," Advances in Artificial Neural Systems, vol. 2011, Article ID 407497, 9 pages, 2011.

[6] T. H. Le, "Applying artificial neural networks for face recognition," Advances in Artificial Neural Systems, vol. 2011, Article ID 673016, 16 pages, 2011.

[7] Y. Xia, "An extended projection neural network for constrained optimization," Neural Computation, vol. 16, no. 4, pp. 863-883, 2004.

[8] Y. Xia and J. Wang, "A recurrent neural network for solving nonlinear convex programs subject to linear constraints," IEEE 
Transactions on Neural Networks, vol. 16, no. 2, pp. 379-386, 2005.

[9] T. Voegtlin, "Recursive principal components analysis," Neural Networks, vol. 18, no. 8, pp. 1051-1063, 2005.

[10] J. Qiu, H. Wang, J. Lu, B. Zhang, and K.-L. Du, "Neural network implementations for PCA and its extensions," ISRN Artificial Intelligence, vol. 2012, Article ID 847305, 19 pages, 2012.

[11] H. Liu and J. Wang, "Integrating independent component analysis and principal component analysis with neural network to predict Chinese stock market," Mathematical Problems in Engineering, vol. 2011, Article ID 382659, 15 pages, 2011.

[12] F. L. Luo, R. Unbehauen, and A. Cichocki, "A minor component analysis algorithm," Neural Networks, vol. 10, no. 2, pp. 291-297, 1997.

[13] G. H. Golub and C. F. Van Loan, Matrix Computations, vol. 3 of Johns Hopkins Series in the Mathematical Sciences, Johns Hopkins University Press, Baltimore, Md, USA, 1983.

[14] C. Chatterjee, V. P. Roychowdhury, J. Ramos, and M. D. Zoltowski, "Self-organizing algorithms for generalized eigendecomposition," IEEE Transactions on Neural Networks, vol. 8, no. 6, pp. 1518-1530, 1997.

[15] H. Kakeya and T. Kindo, "Eigenspace separation of autocorrelation memory matrices for capacity expansion," Neural Networks, vol. 10, no. 5, pp. 833-843, 1997.

[16] Y. Liu, Z. You, and L. Cao, "A functional neural network for computing the largest modulus eigenvalues and their corresponding eigenvectors of an anti-symmetric matrix," Neurocomputing, vol. 67, no. 1-4, pp. 384-397, 2005.

[17] Y. Liu, Z. You, and L. Cao, "A functional neural network computing some eigenvalues and eigenvectors of a special real matrix," Neural Networks, vol. 18, no. 10, pp. 1293-1300, 2005.

[18] Y. Liu, Z. You, and L. Cao, "A recurrent neural network computing the largest imaginary or real part of eigenvalues of real matrices," Computers \& Mathematics with Applications, vol. 53, no. 1, pp. 41-53, 2007.

[19] E. Oja, "Principal components, minor components, and linear neural networks," Neural Networks, vol. 5, no. 6, pp. 927-935, 1992.

[20] R. Perfetti and E. Massarelli, “Training spatially homogeneous fully recurrent neural networks in eigenvalue space," Neural Networks, vol. 10, no. 1, pp. 125-137, 1997.

[21] L. Xu, E. Oja, and C. Y. Suen, "Modified Hebbian learning for curve and surface fitting," Neural Networks, vol. 5, no. 3, pp. 441457, 1992.

[22] Q. Zhang and Y. W. Leung, "A class of learning algorithms for principal component analysis and minor component analysis," IEEE Transactions on Neural Networks, vol. 11, no. 2, pp. 529$533,2000$.

[23] Y. Zhang, Y. Fu, and H. J. Tang, "Neural networks based approach for computing eigenvectors and eigenvalues of symmetric matrix," Computers \& Mathematics with Applications, vol. 47, no. 8-9, pp. 1155-1164, 2004.

[24] L. Mirsky, "On the minimization of matrix norms," The American Mathematical Monthly, vol. 65, pp. 106-107, 1958.

[25] C. P. ] Huang and R. T. Gregory, A Norm-Reducing Jacobi-Like Algorithm for the Eigenvalues of Non-Normal Matrices, Colloquia Mathematica Societatis Janos Bolyai, Keszthely, Hungary, 1977.

[26] I. Kiessling and A. Paulik, "A norm-reducing Jacobi-like algorithm for the eigenvalues of non-normal matrices," Journal of Computational and Applied Mathematics, vol. 8, no. 3, pp. 203207, 1982.
[27] R. Sacks-Davis, "A real norm-reducing Jacobi-type eigenvalue algorithm," The Australian Computer Journal, vol. 7, no. 2, pp. 65-69, 1975. 


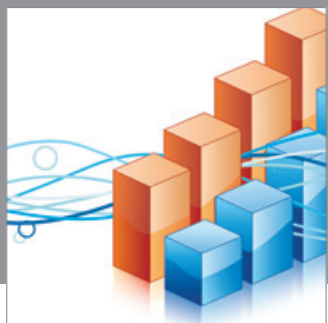

Advances in

Operations Research

mansans



The Scientific World Journal
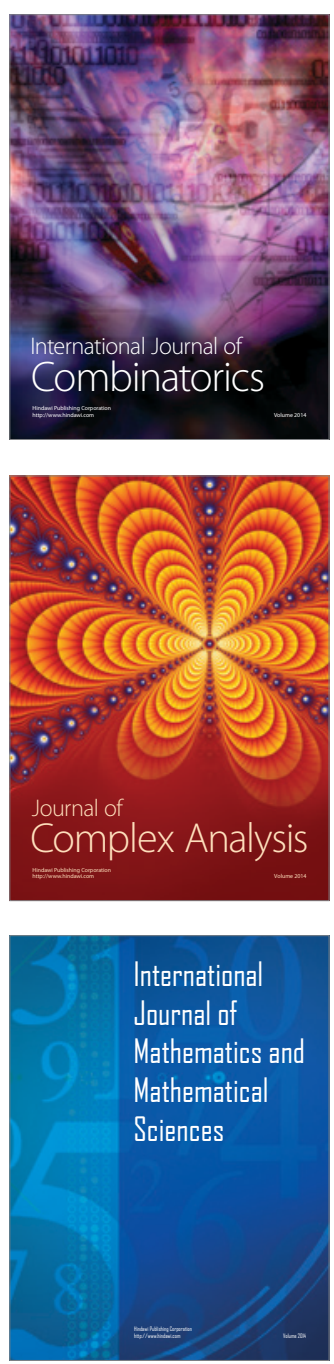
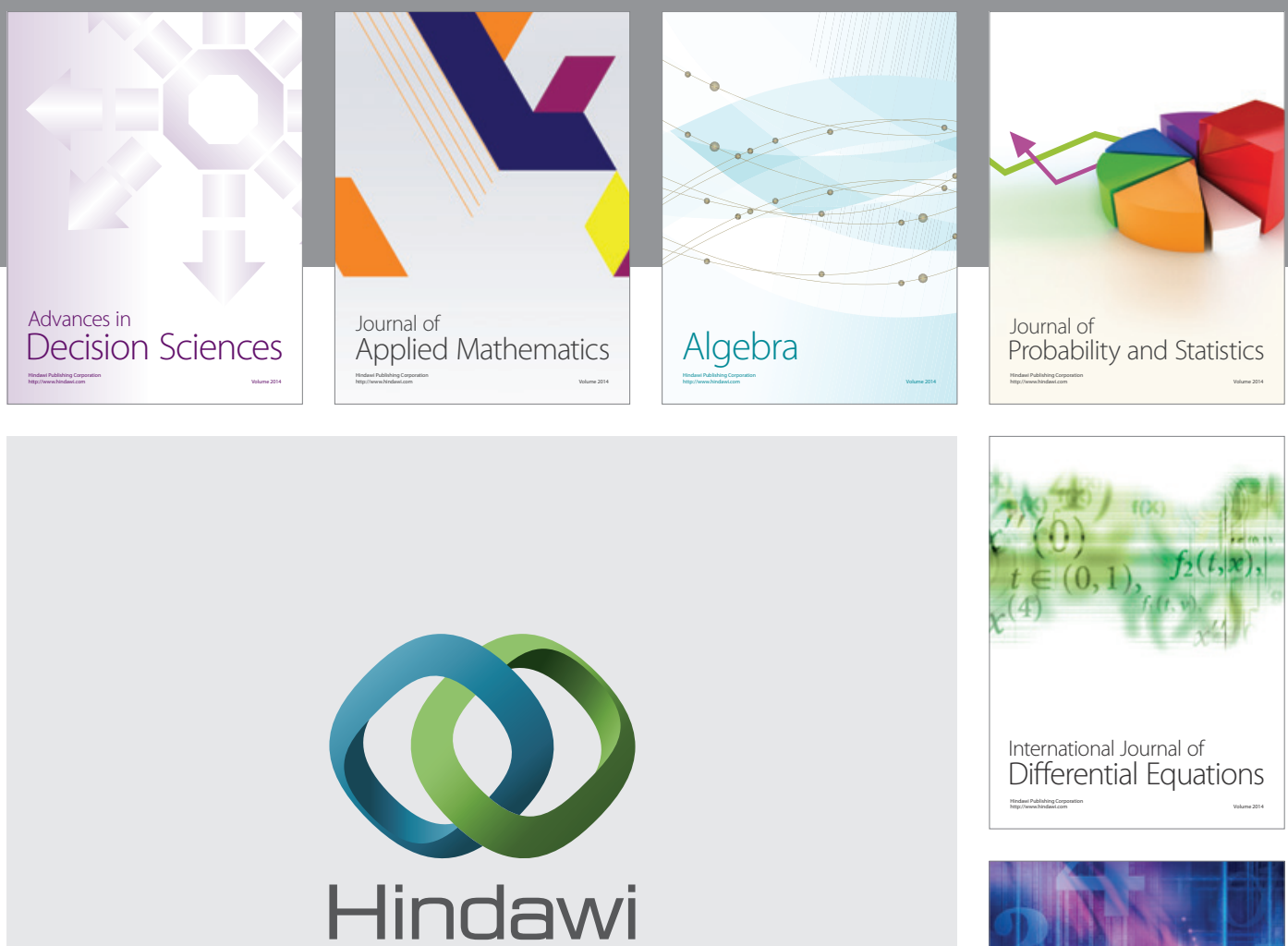

Submit your manuscripts at http://www.hindawi.com
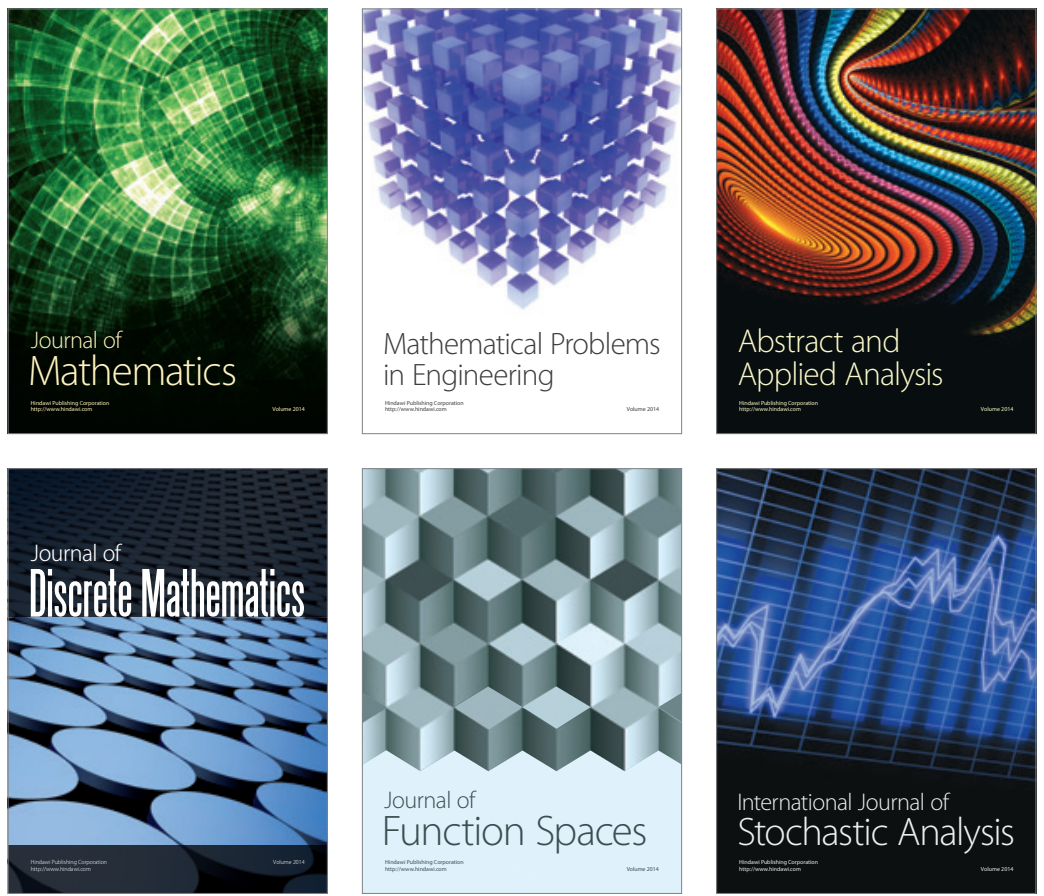

Journal of

Function Spaces

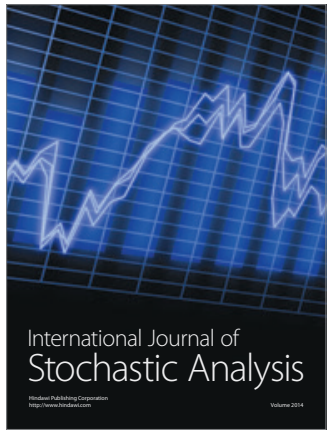

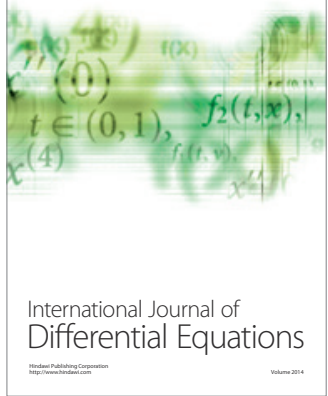
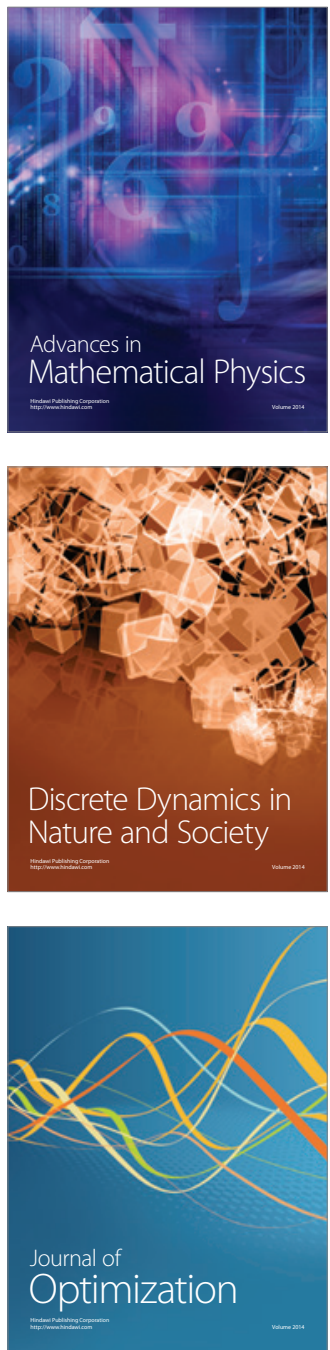